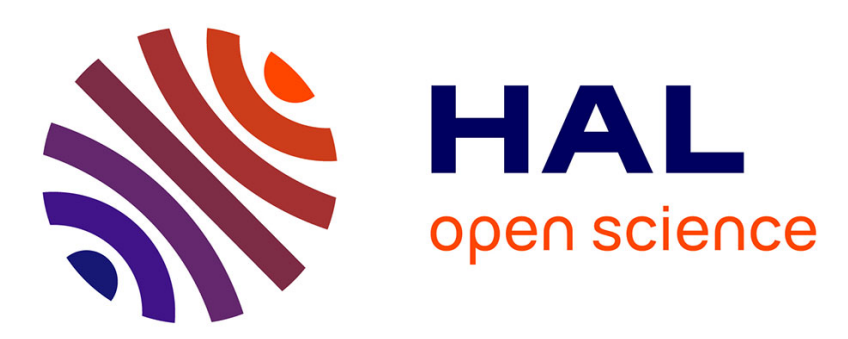

\title{
Le dîner des Français : un synchronisme alimentaire qui se maintient
}

\author{
Thibaut De Saint Pol
}

\section{To cite this version:}

Thibaut De Saint Pol. Le dîner des Français : un synchronisme alimentaire qui se maintient. Economie et Statistique / Economics and Statistics, 2007, 400, pp.45-69. halshs-00145629

\section{HAL Id: halshs-00145629 \\ https://shs.hal.science/halshs-00145629}

Submitted on 11 May 2007

HAL is a multi-disciplinary open access archive for the deposit and dissemination of scientific research documents, whether they are published or not. The documents may come from teaching and research institutions in France or abroad, or from public or private research centers.
L'archive ouverte pluridisciplinaire HAL, est destinée au dépôt et à la diffusion de documents scientifiques de niveau recherche, publiés ou non, émanant des établissements d'enseignement et de recherche français ou étrangers, des laboratoires publics ou privés. 


\section{Le dîner des Français : un synchronisme alimentaire qui se maintient}

\section{Thibaut de Saint Pol*}

Les enquêtes Emploi du Temps réalisées par l'Insee en 1986 et en 1998 permettent de s'interroger sur la place consacrée au dîner dans la soirée des Français et sur la manière dont il s'inscrit au sein des autres activités. Le repas du soir, présent en activité principale dans la plupart des carnets remplis par les enquêtés, a lieu à peu près dans la même période pour l'ensemble des individus : on observe en France une forte synchronisation des pratiques alimentaires.

En comparant les séquences d'activités qui composent les soirées, des similitudes apparaissent, tant du point de vue du dîner que des activités qui l'entourent, et il est ainsi possible de distinguer plusieurs types d'organisation de cette période, propres à certains groupes sociaux. Les soirées des femmes se différencient ainsi fortement de celles des hommes par l'importance des travaux ménagers qu'elles réalisent autour du dîner. La présence plus ou moins grande de la télévision semble aussi déterminer l'heure de ce repas. Les plus âgés dînent généralement plus tôt que les autres, comme les moins diplômés et les individus aux plus bas revenus. L'influence du jour de la semaine apparaît également, avec une place plus importante consacrée aux sorties et des dîners qui durent plus longtemps le samedi soir.

De plus, l'organisation de la soirée des Français et la place du dîner en son sein demeurent très proches entre 1986 et 1998. Les comportements ont peu évolué. Contrairement à ce que supposent les discours sur la déstructuration des repas traditionnels, cette étude fait apparaître la grande stabilité de la séquence du dîner et l'importance du temps alimentaire aujourd'hui en France.

\footnotetext{
* Thibaut de Saint Pol est membre du laboratoire de sociologie quantitative du Centre de recherche en économie et statistique (Crest) de l'Insee et appartient également à l'Observatoire sociologique du changement (FNSP,CNRS).

L'auteur remercie Alain Chenu, Jacques Siracusa et Laurent Lesnard, ainsi que deux relecteurs anonymes dont les remarques et suggestions ont contribué à améliorer cet article.
} 
$\mathbf{L}$ e repas est un poste essentiel de l'emploi du temps des individus et un certain nombre de pratiques sociales se greffent autour de ces prises alimentaires. Dîner chez soi ou à l'extérieur, seul ou en compagnie de son conjoint ou d'un ami sont autant de déclinaisons d'une pratique fortement marquée par les habitudes et par l'âge. Le caractère vital de l'alimentation amène chaque individu à réserver une partie de son capital temporel quotidien pour s'alimenter et cette activité figure dans tous les emplois du temps. Mais du sandwich avalé en quelques minutes à la réception formelle qui dure plusieurs heures, chaque repas est modulé par les contraintes sociales, spatiales et temporelles dans lesquelles il s'inscrit.

Lorsque l'idée d'enquêter sur l'organisation quotidienne des activités a été développée (1), la France se distinguait par la place accordée aux trois repas (petit déjeuner, déjeuner et dîner) et leur concentration temporelle pour une grande partie des individus: les Français déjeunaient et dînaient globalement durant les mêmes intervalles de temps (Szalai, 1972). De plus, l'alimentation des Français se caractérisait par une grande régularité des prises alimentaires pour l'ensemble de la population.

Cette situation contrastait avec celle d'autres pays, comme les États-Unis pour lesquels Fischler notait dès la fin des années 1970 la quasi-disparition du repas comme moment partagé par l'ensemble des membres d'un ménage et parlait même de " gastro-anomie » (Fischler, 1979). Cette déstructuration des repas portait sur le déroulement du repas et sa composition, mais aussi sur sa régularité. Les mêmes tendances s'observaient en Europe et le synchronisme alimentaire français semblait lui aussi condamné.

Cette particularité des prises alimentaires n'est toutefois pas sans importance puisqu'elle a des conséquences directes sur le corps et la santé. Les Français, et en particulier les Françaises, ont la corpulence moyenne la plus faible d'Europe (de Saint Pol, 2006). Or, Fischler (1996) relève par exemple qu'il est très vraisemblable que le caractère " réglé » de notre alimentation joue un rôle dans la minceur des Français et dans leur faible taux de maladies coronariennes, notamment en limitant la multiplication d'épisodes alimentaires en dehors des repas principaux qui jouent un grand rôle dans la prise de poids (Basdevant et al., 1993).

Ce caractère « réglé » renvoie à l'existence d'un modèle alimentaire composé de trois principaux repas pendant lesquels se concentre l'essentiel des prises alimentaires et constitue une caractéristique importante de l'emploi du temps des Français. On ne retrouve par exemple rien de similaire au Royaume-Uni (cf. graphique I). Si l'on observe trois légers pics, beaucoup moins d'individus mangent au même moment et on ne peut pas véritablement parler de synchronisme alimentaire. En France, bien que les activités qui composent leur emploi du temps soient mul-

1. Programme dirigé par Szalai en 1965-66 qui portait sur des échantillons représentatifs des populations urbaines de douze pays.

Graphique I
Les plages alimentaires au Royaume-Uni et en France

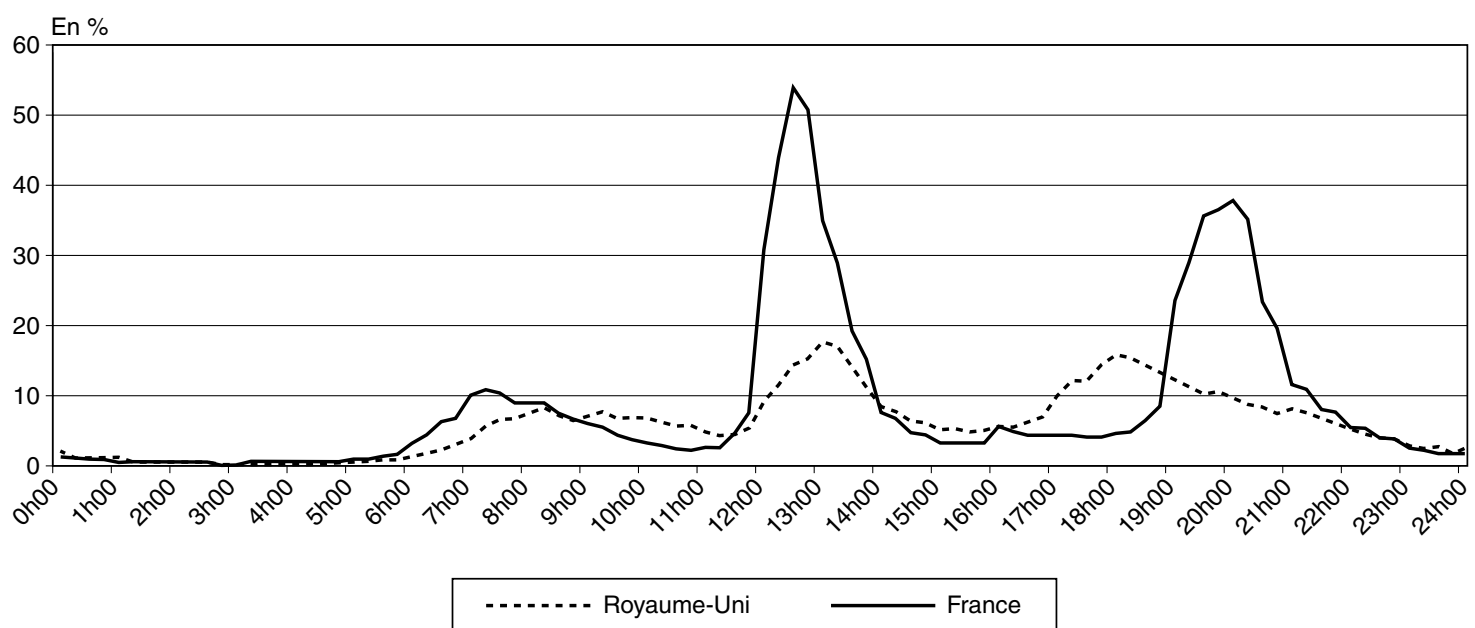

Lecture : au Royaume-Uni, 17,6 \% des individus inscrivent une activité alimentaire à 13 heures contre 54,1\% en France à $12 h 30$. Source : Time Use Survey 2000, ONS, et enquête Emploi du temps 1998-1999, Insee. 
tiples, les individus s'alimentent massivement durant les mêmes plages horaires.

Cette synchronisation est d'autant plus importante que si l'on ajoute les temps de travail domestique qui y sont directement liés (cuisine, vaisselle, etc.), manger est une des activités principales de la journée, du réveil au coucher. L'alimentation est une contrainte qui s'impose à l'individu (pour survivre il faut manger) et sert de cadre à la journée en définissant les espaces temporels distincts que sont la matinée, l'aprèsmidi ou encore la soirée. Mais elle est en même temps contrainte par les autres activités qui l'entourent (le journal télévisé commence par exemple à $20 \mathrm{~h} 00$, etc.). Dans cette perspective, la place du repas au sein de la séquence des activités qui l'encadrent apparaît déterminante.

\section{Le pic des dîners : un synchronisme alimentaire qui se situe autour de $20 \mathrm{h00}$}

Depuis les années 1980, les études portant sur l'alimentation et les temps sociaux s'intéressent à ce que Aymard et al. (1993) appellent un «leitmotiv de la modernité alimentaire»: la thèse de la «crise » des repas traditionnels et de leur «déstructuration ». Le « modèle » alimentaire français serait ainsi en voie de dispa- rition, notamment du fait de l'augmentation de l'alimentation hors repas. Si l'alimentation ne joue plus le même rôle qu'au lendemain de la Seconde Guerre mondiale et si «une dévaluation progressive des pratiques alimentaires » a eu lieu au cours des années 1980 (Pynson, 1987), plusieurs études (Grignon, 1987, 1998 ; Herpin, 1988) témoignent plutôt du maintien de la grille des repas quotidiens. Ainsi, comme l'observe également Larmet (2002), les enquêtes Emploi du temps de 1986 et de 1998 (cf. encadré 1) font apparaître une forte synchronisation des pratiques alimentaires des Français autour de trois pics quotidiens. Les repas traditionnels, au premier rang desquels se trouve le dîner, constituent encore l'essentiel du mode d'alimentation pour l'ensemble de la population.

Le repas du soir occupe cependant une place particulière au sein des repas quotidiens (de Saint Pol, 2005a). Il est moins soumis au travail professionnel que le déjeuner, qui s'inscrit généralement dans la journée de travail et dont les horaires sont souvent imposés, si ce n'est par l'entreprise, du moins par le rythme quotidien de travail. Il est par ailleurs soumis à des contraintes temporelles plus diverses que le petit déjeuner, encadré en semaine par l'heure du lever et l'heure de départ du domicile et s'inscrivant moins souvent pour les individus dans le

Encadré 1

\section{L'ENQUÊTE EMPLOI DU TEMPS DE 1998}

L'enquête Emploi du temps de 1998 a été réalisée par I'Insee auprès de 8000 ménages. Elle s'inscrit dans la lignée des enquêtes du même type réalisées en 1966 , 1974 et 1986 et repose sur le remplissage d'un carnet journalier. Chaque enquêté y note ses activités tout au long de la journée en indiquant leur nature, leur début et leur fin. Le carnet recouvre ainsi une journée de 0 h00 à 24 h00 découpée en plages horaires de 10 minutes. Si plusieurs activités sont réalisées en même temps, seules les deux principales sont retenues. L'une est codée en activité principale et l'autre en activité secondaire. La présentation du carnet journalier de 1998 suggère à l'enquêté d'opérer lui-même cette distinction. II doit également renseigner le lieu, le trajet, en présence de qui et dans quel but est effectuée l'activité (personnel, professionnel, pour un autre ménage, associatif).

L'enquête Emploi du temps a été engagée sur un an afin d'éviter toute saisonnalité et que tous les jours de la semaine soient représentés. L'échantillon a été choisi au moyen d'un tirage par sondage de 12000 logements à partir du recensement de la population de 1990. Tous les individus du ménage âgés de plus de quinze ans sont alors consultés sur leurs emplois du temps et 15400 carnets ont été ainsi recueillis. Dans un souci de comparaison des enquêtes et de mise en œuvre de la méthode choisie, cette étude se limite aux individus de communes urbaines âgés de 18 à 64 ans soit 8257 individus en 1998 et 9975 en 1986. L'alimentation apparaît dans presque tous les carnets, avec une durée plus ou moins longue. Seuls 17 individus n'ont mentionné aucune plage de repas en activité principale.

Ces carnets sont marqués par la richesse de l'information qu'ils contiennent, mais en même temps par l'hétérogénéité de cette information (une multitude de détails et précisions pour certains et la seule inscription des principales activités pour d'autres). Le nombre de lignes des carnets varie en effet entre 7 et 63 pour une médiane de 22. Comparer ces données demande donc un certain nombre de précautions. Par ailleurs, les variables correspondant à la durée de certaines de ces activités sont marquées par la présence d'un mode égal à 0 , comme les cérémonies religieuses ou la présence dans un cimetière. II est en outre difficile de savoir si les activités décrites dans les carnets sont réalisées de manière quotidienne ou exceptionnelle puisque l'on ne dispose que d'une observation de vingt-quatre heures par individu. 
temps familial (Marenco, 1992). Le dîner est le repas le plus pris au domicile (Volatier, 1999) et apparaît ainsi comme un des derniers refuges de la sociabilité familiale (Herpin, 1988). Les enquêtes Emploi du temps de 1986 et 1998 font toutes deux apparaître une concentration des prises alimentaires entre $19 \mathrm{~h} 00$ et $21 \mathrm{~h} 00$ (cf. graphique II). Le pic se situe en 1998 juste après $20 \mathrm{~h} 00$, c'est-à-dire un peu plus tard qu'en 1986 , avec $38 \%$ de la population qui effectue une activité de type alimentaire.

Cette concordance entre les emplois du temps témoigne du caractère social de l'organisation du temps de repas. Le temps alimentaire est à la rencontre de plusieurs temps sociaux. La décision de dîner à une certaine heure ne dépend pas uniquement du rythme biologique et en particulier de la perception de la faim. Les prises alimentaires s'inscrivent au sein d'autres activités et de leurs contraintes temporelles. Comprendre le repas des Français, c'est aussi s'interroger sur la manière dont cette activité s'inscrit dans la séquence des autres activités. En se concentrant plus particulièrement sur la période qui correspond au pic des dîners (18h50-21h30), soit 16 périodes de dix minutes chacune, cette étude se propose de chercher à comprendre comment le dîner s'inscrit dans la soirée des individus ayant répondu à l'enquête Emploi du temps 1998-1999 (cf. encadré 1).

Les données permettant de s'intéresser à cet objet sont peu nombreuses et souffrent souvent de trop de défauts pour que leurs résultats soient exploitables, en particulier, lorsque l'on demande aux individus de se rappeler et d'éva-

\section{Graphique I}

\section{Le pic des dîners}

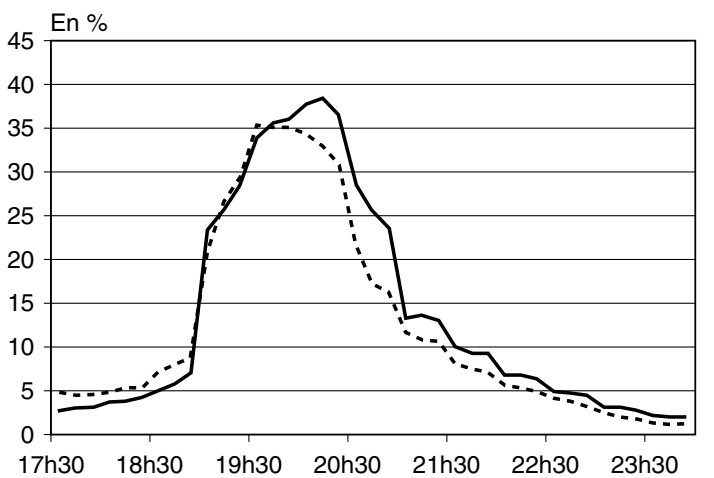

$$
-1998
$$

Lecture : en 1998, à 19h30, 34,5\% des individus déclarent une activité alimentaire.

Source : enquête Emploi du temps 1998-1999, Insee. luer globalement le temps qu'ils ont consacré la veille à l'alimentation ou bien lorsqu'on leur demande combien ils ont effectué de prises alimentaires. Lors de l'enquête Emploi du temps, chaque individu interrogé a inscrit l'ensemble de ses activités quotidiennes, et en particulier les épisodes alimentaires. Chacune des activités qu'il a inscrites sur le carnet journalier peut être considérée comme un élément d'une séquence d'activités (cf. encadré 2). L'enquête Emploi $d u$ temps constitue ainsi un type de données très original pour s'intéresser à la question de la « déstructuration » des repas.

L'utilisation d'une technique nouvelle de traitement séquentiel des données, l'appariement optimal (cf. encadré 3), permet de mettre en évidence des types de séquences et d'étudier l'organisation temporelle de ce moment de la journée. Sa mise en œuvre sur les données de l'enquête Emploi du temps 1998-1999 conduit à un regroupement des séquences en dix classes, découpage optimal du point de vue de l'homogénéité des groupes. Le nombre de dix classes peut sembler élevé, mais il répond à une volonté d'accéder à des comportements singuliers même s'ils sont en petit nombre. Les effectifs des groupes sont assez différents et certains témoignent de pratiques plus minoritaires que d'autres (cf. tableau 1). Ainsi, la classe la plus grande (la neuvième avec 2021 séquences) regroupe six fois et demie plus de séquences que la plus petite (la classe 10 qui n'en compte que 303). La réalisation du même traitement sur les données de l'enquête Emploi du Temps de 1986 permet de se pencher sur l'évolution de ces pratiques. Les dix classes construites pour 1986 ne correspondent pas parfaitement à celles obtenues pour 1998, toutefois les similitudes sont assez importantes pour que les groupes construits aux deux dates puissent être rapprochés.

Cette étude privilégie deux instruments de représentation des groupes issus de l'appariement. Le premier est la séquence moyenne de la classe, séquence dont la distance à l'ensemble des autres séquences du groupe est minimale et qui vise à saisir les contrastes entre classes. En d'autres termes, c'est la séquence qui est la plus proche de toutes les autres : elle est au centre de la classe et constitue ainsi un bon résumé des éléments constitutifs du groupe. L'intérêt de ce premier outil est de conserver un raisonnement en séquences, mais aussi de s'assurer que la description qui est faite de la classe correspond bien à la réalité : il existe au moins un individu dans notre base de données qui a inscrit dans son carnet l'enchaînement d'activités qui correspond à la séquence moyenne. 
$\mathrm{Au}$ centre des groupes se trouvent en 1998 des comportements très marqués puisque que trois séquences moyennes sont constituées d'une seule activité se prolongeant sur toute la période (cf. tableau 2). En effet, la séquence moyenne de la classe 6 ne contient que l'activité «Travail-Études », celle de la classe 7 l'activité «Repas», et celle de la classe 8
«Rencontres-sorties ». Ce résultat est une conséquence directe du protocole de regroupement des séquences et une preuve qu'il a bien fonctionné. Autour de ces comportements très marqués gravitent un certain nombre de déclinaisons de cette soirée-type que la séquence moyenne, qui donne juste une tendance, ne nous permet pas d'apprécier.

\section{Encadré 2}

\section{L'INTÉRÊT DE L'ANALYSE DES EMPLOIS DU TEMPS EN TERMES DE SÉQUENCES}

L'enquête Emploi du Temps de l'Insee se prête très bien à la lecture en termes de séquences. En effet, les données recueillies dans le carnet journalier se présentent initialement de manière séquentielle : la journée est divisée en 144 intervalles de 10 minutes pour l'enquête de 1998. Ces intervalles correspondent à 144 variables $a_{i}$ qui contiennent le code de l'activité réalisée par l'enquêté pendant ces dix minutes. Ainsi, la variable $a_{1}$ concerne l'activité pratiquée entre 0 h00 et 0 h10 (qui est le sommeil dans plus de $85 \%$ des cas...). On a donc pour chaque individu une séquence de 144 éléments qui traduit directement l'emploi du temps noté sur le carnet journalier. Ce sont donc des données assez originales d'un point de vue statistique puisque chacune des 144 variables entretient un lien chronologique implicite avec les autres $a_{i}$. Elles imposent de raisonner autant en termes de variables qualitatives que de séries temporelles, ce qui rend ces données délicates à exploiter avec les outils statistiques classiques et une lecture en termes de moyenne et de durée est souvent privilégiée (Larmet (2002), sur les temps consacrés à la sociabilité alimentaire).

S'il est intéressant pour comprendre l'organisation du dîner de faire une lecture de la soirée en termes de processus, il est impossible d'observer les régularités entre individus sur les milliers de séquences contenues dans les carnets journaliers. Ce point, qui est central pour l'analyse des dynamiques des emplois du temps, ne peut être saisi aisément avec les outils statistiques classiques. Les modèles en termes de durée par exemple, fréquemment utilisés dans des cas similaires, ne sont pas adaptés car ils ne prennent en compte que l'information passée. Or, les emplois du temps et la place du repas en leur sein se construisent également en fonction des activités futures.

Les comparaisons au moyen d'instruments statistiques classiques entre l'enquête Emploi du temps de 1986 et celle de 1998 sont par ailleurs très délicates, en particulier à cause du changement de cinq à dix minutes de l'intervalle minimal entre deux activités dans le carnet journalier. Cette modification induit une perte de précision importante pour le travail domestique en général, plus fractionné que les autres activités et peut influer directement sur la durée des repas. Les individus peuvent par exemple reporter une tâche domestique courte (mettre la table par exemple) sur la durée du repas. II y a donc la possibilité d'apparition de biais méthodologiques non négligeables dans les comparaisons classiques entre les deux enquêtes. Les méthodes d'appariement optimal (M. A. O.), en utilisant l'enchaînement des activités plutôt que les durées, permettent de diminuer l'importance de ces éventuels biais. En ne retenant qu'un intervalle de cinq minutes sur deux dans les séquences de 1986, on y réduit la place des activités les plus courtes, moins présentes dans les intervalles de dix minutes de 1998.

Tableau 1

Résumé de la classification

\begin{tabular}{|c|c|l|}
\hline Classe & $\begin{array}{c}\text { Effectif } \\
\text { (en } \%)\end{array}$ & \multicolumn{1}{|c|}{ Description sommaire des groupes en 1998} \\
\hline 1 & 5,8 & Un repas encadré de tâches ménagères \\
\hline 2 & 12,0 & Un repas en première moitié de période, suivi par une multitude d'activités \\
\hline 3 & 7,5 & Un repas en seconde moitié de période, encadré de tâches ménagères \\
\hline 4 & 20,8 & Une soirée découpée entre le repas et la télévision \\
\hline 5 & 5,4 & Une soirée entièrement consacrée à la télévision \\
\hline 6 & 9,4 & L'alimentation s'insère dans le temps de travail \\
\hline 7 & 6,0 & Une prépondérance des plages alimentaires \\
\hline 8 & 4,9 & Un repas qui s'insère dans des pratiques de loisirs à l'extérieur \\
\hline 9 & 24,5 & Un repas en seconde moitié de période, après une multitude d'activités qui se prolongent jusqu'à 20h00. \\
\hline 10 & 3,7 & Des individus qui se couchent plus tôt que les autres. \\
\hline
\end{tabular}

Champ : la plage horaire considérée débute à 18 h50 et finit à 21 h30.

Source : enquête Emploi du temps 1998-1999, Insee. 
Le second instrument donne au contraire le comportement de l'ensemble des individus de la classe dans un graphique présentant, sur chaque plage horaire de dix minutes, la répartition de l'ensemble des activités réalisées par les individus du groupe dans neuf grands types d'activités, une ou deux se distinguant nettement selon les classes, signe que celles-ci sont homogènes (cf. annexe 3). Le traitement séquentiel permet ainsi de regrouper des individus selon la proximité de leur emploi du temps et chacun des dix groupes fait apparaître un type d'organisation de la période. Le repas n'a en aucun cas été privilégié sur les autres activités dans la construction des classes et il est possible de présenter chacun de ces types en se penchant sur la place du dîner en son sein.

\section{Des types féminins d'organisation encore marqués par la place des travaux ménagers}

Parmi les groupes issus de l'appariement optimal, il en est trois dans l'emploi du temps des-

Encadré 3

\section{LE PRINCIPE DES MÉTHODES D'APPARIEMENT OPTIMAL}

Empruntées aux biologistes moléculaires, les méthodes d'appariement optimal (Optimal Matching Analysis (O.M.A.)) servaient à l'origine à étudier des séquences d'ADN. Elles furent appliquées pour la première fois en sociologie sur des problèmes tels que l'État providence, pour analyser l'ordre des séquences correspondant aux différents programmes ou décisions intervenues dans différents pays, ou la carrière des musiciens (Abbott, 1995 ; Abbott et Hrycak, 1990). Ces méthodes permettent de comparer des séquences entre elles et de rassembler les plus proches. La comparaison s'effectue sans avantager aucun des éléments qui composent les séquences. Autrement dit, le traitement séquentiel ne privilégie pas dans notre cas le repas sur les autres activités et ne donne donc pas lieu à l'édification de classes dont le centre d'intérêt aurait biaisé la construction. Mais un des principaux intérêts de la méthode se situe dans la prise en compte de toutes les dimensions temporelles de l'emploi du temps. Un mode de comparaison plage horaire par plage horaire, bien que beaucoup plus aisé à mettre en place, ne permet pas de saisir la dimension horizontale des séquences : deux emplois du temps décalés de seulement dix minutes, soit un élément, apparaissent alors comme totalement différents. Cette technique permet justement de prendre en compte de tels décalages dans la comparaison des séquences.

Ce groupe de méthodes vise à se donner un moyen de comparer puis de regrouper un grand nombre de séquences (ADN ou emploi du temps). Ces deux mouvements correspondent aux deux grandes étapes de cette technique: la procédure de minimisation et la méthode de classification.

Pour construire une distance, il s'agit de mettre en place une procédure de minimisation. La finalité de cette première étape est d'arriver à déterminer pour chaque couple de séquences comment on peut passer de l'une à l'autre le plus facilement possible, c'est-àdire, en termes mathématiques, pour le moindre coût. On va considérer toutes les façons de transformer une première séquence en une seconde et on retiendra la plus simple. Pour passer d'une séquence à une autre, on peut utiliser trois opérations élémentaires : l'insertion, la suppression et la substitution d'un élément en un autre. Ces trois opérations sont celles qu'utilisent intuitivement les individus pour composer et gérer leur emploi du temps au fil de la journée. Ils suppriment et ajoutent des activités. Ils en décalent d'autres. Deux emplois du temps semblables mais décalés de dix minutes doivent apparaître très proches. Deux emplois du temps qui ne diffèrent que d'une activité présente dans l'un et non dans l'autre le sont aussi. Ce sont ces dimensions que les méthodes d'appariement optimal cherchent à saisir.

Pour comparer deux séquences, on systématise cette approche en considérant toutes les manières de transformer la première séquence en la seconde au moyen de suppressions, insertions et substitutions. Chacune de ces transformations d'une séquence en une autre possède un coût différent, calculé en fonction de la nature des données. On calcule la distance entre deux séquences comme le coût minimum pour passer de la première à la seconde. Cette procédure de minimisation permet d'obtenir une distance pour chaque paire de séquences. II ne reste alors plus qu'à mettre en œuvre des techniques de classification pour regrouper les séquences en fonction de la distance construite précédemment. C'est la méthode bêta-flexible qui a été utilisée ici (Lesnard et de Saint Pol (2006) et Lesnard (2006), pour plus de précisions). Les coûts des trois opérations élémentaires ont été déterminés en termes de fréquence des différents éléments constitutifs des séquences. En particulier, à chaque opération de substitution a été affectée sa probabilité de passage : le coût de la substitution d'un repas par un épisode de télévision est la probabilité sur l'ensemble des séquences qu'une période de dix minutes de télévision suive dix minutes de repas (de Saint Pol (2005b), pour plus de précisions).

L'algorithme des méthodes d'appariement optimal restant assez simple à mettre en œuvre, on peut sans trop de difficulté écrire soi-même un programme réalisant la procédure de minimisation. II existe également deux principaux logiciels libres qui permettent de réaliser ces traitements. Les résultats présentés dans cet article ont été réalisés avec le programme de l'Université de Bochum, TDA 6.2, qui a été développé spécifiquement pour traiter les séquences temporelles. 
quels les tâches ménagères et familiales occupent en 1998 une grande place (cf. tableau 3). L'organisation traditionnelle des repas est toujours actuelle, puisque ces soirées-types sont essentiellement féminines. En 1999, les deux tiers du travail domestique sont toujours effectués par les femmes (Brousse, 1999). Ainsi les populations des première et troisième soiréetypes consacrent en moyenne respectivement 53 et 63 minutes aux tâches ménagères sur la période étudiée et se composent toutes deux de près de $80 \%$ de femmes (cf. tableau 4). La deuxième classe compte quant à elle près de $65 \%$ de femmes. Le travail domestique apparaît ainsi comme une constante des emplois du temps féminins.

Pour autant, les trois soirées-types féminines correspondent à trois modes de vie totalement différents. Ainsi le repas des individus de la première classe est généralement pris en milieu de période et donne lieu à de très fortes concentrations ( $79 \%$ de la population à $20 \mathrm{~h} 10)$. Il dure aussi plus longtemps en moyenne que dans la plupart des autres groupes (48 minutes). Mais la particularité de ce groupe tient surtout à la place occupée par les tâches ménagères qui encadrent le repas et qui lui sont sans doute en partie liées ( $82 \%$ déclarent une activité de type «cuisine-linge-ménage » à 19h10, soit une heure avant le pic du repas). À elles seules, alimentation et tâches ménagères occupent au moins $80 \%$ de la population sur chaque plage horaire entre $19 \mathrm{~h} 10$ à $20 \mathrm{~h} 30$, pour même atteindre $99 \%$ à 19 h50. Le repas constitue donc pour ces individus une des occupations principales de la soirée. Si la plupart des activités sont représentées à $18 \mathrm{~h} 50$, toutes subissent une baisse notable avec l'entrée dans la période du repas. À l'inverse, la période après le dîner est principalement consacrée à la télévision, qui apparaît après $20 \mathrm{~h} 30$ avec le film ou le programme de

Tableau 2

Séquences-moyennes des groupes de 1998 (1)

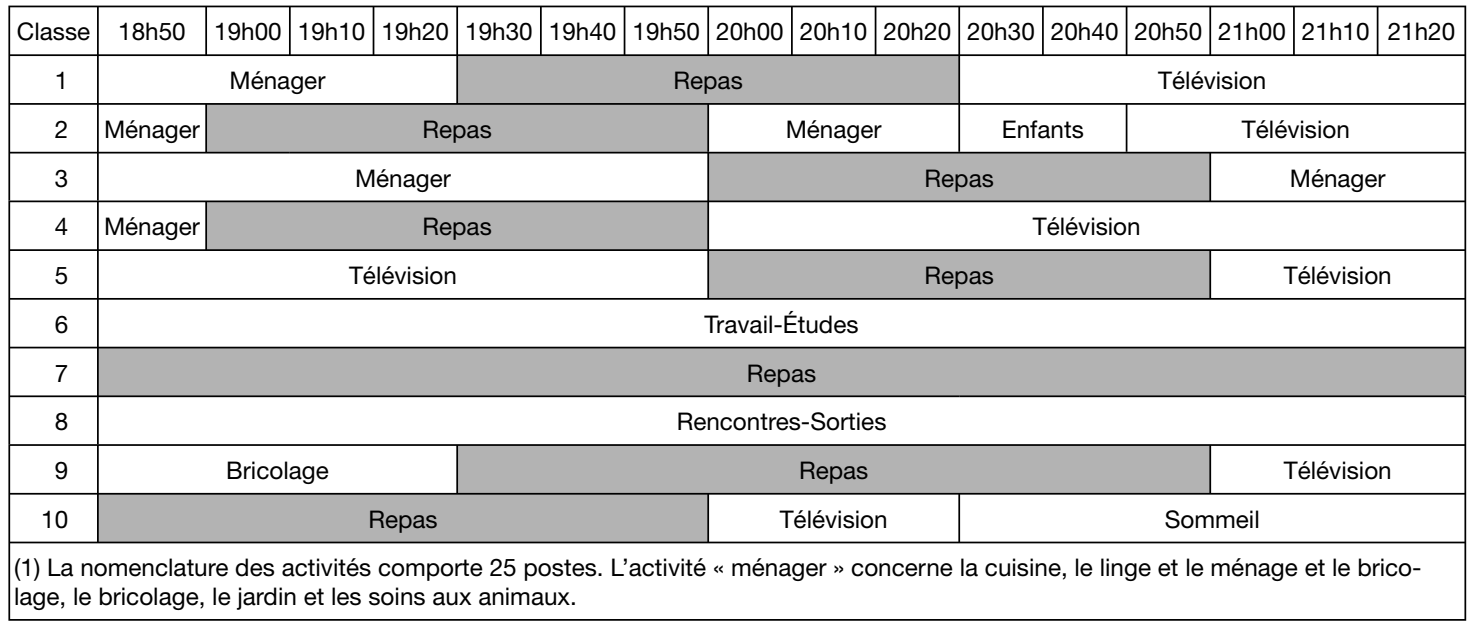

Lecture : à 18h50, l'individu au centre de la première classe a inscrit une activité de type "cuisine-linge-ménage ». Source : enquête Emploi du temps 1998-1999, Insee.

Tableau 3

Les activités entre $18 \mathrm{~h} 50$ et $21 \mathrm{~h} 30$ en 1998

\begin{tabular}{|c|c|c|c|c|c|c|c|c|c|c|c|}
\hline \multirow{2}{*}{ Activités } & \multicolumn{10}{|c|}{ Classe } & \multirow{2}{*}{ Ensemble } \\
\hline & 1 & 2 & 3 & 4 & 5 & 6 & 7 & 8 & 9 & 10 & \\
\hline Alimentation & 48 & 42 & 40 & 37 & 43 & 26 & 113 & 29 & 36 & 43 & 42 \\
\hline Cuisine-linge-ménage & 53 & 25 & 63 & 14 & 14 & 7 & 8 & 8 & 13 & 12 & 19 \\
\hline Télévision & 46 & 20 & 9 & 88 & 84 & 11 & 11 & 11 & 21 & 25 & 37 \\
\hline Loisirs (hors télévision et sorties) & 3 & 29 & 13 & 5 & 5 & 5 & 6 & 4 & 18 & 6 & 12 \\
\hline Sommeil & 0 & 3 & 3 & 1 & 3 & 1 & 0 & 1 & 4 & 54 & 4 \\
\hline Travail-études & 0 & 5 & 3 & 1 & 2 & 86 & 1 & 2 & 7 & 1 & 11 \\
\hline Trajets & 2 & 7 & 5 & 3 & 3 & 14 & 7 & 10 & 15 & 4 & 8 \\
\hline Maison et famille & 7 & 25 & 19 & 9 & 6 & 8 & 10 & 9 & 31 & 14 & 17 \\
\hline Rencontres-spectacles-promenades & 1 & 3 & 3 & 2 & 2 & 1 & 4 & 86 & 16 & 1 & 10 \\
\hline
\end{tabular}

Lecture: les individus contenus dans la première classe consacrent en moyenne 48 minutes à l'alimentation sur la période 18h50$21 h 30$.

Source : enquête Emploi du temps 1998-1999, Insee. 
début de soirée pour regrouper près de $99 \%$ de la population après $21 \mathrm{~h} 00$.

Les individus de ce groupe vivent généralement en couple, souvent avec enfant(s). Les femmes au foyer y sont surreprésentées. Mais on trouve aussi dans cette classe des employé(e)s et du personnel de service (cf. tableau 5). Si le repas est aussi étalé sur la période, c'est que, de sa réalisation à sa consommation effective, il constitue une activité qui incombe à l'épouse ou à la mère et dont dépendent souvent d'autres membres du ménage (conjoints ou enfants) qui consacrent généralement moins de temps à ces tâches ménagères.

La principale différence entre les populations des première et deuxième classes tient à l'âge de leurs membres. Les femmes qui compo- sent majoritairement la deuxième classe sont plus jeunes. À l'inverse, plus un individu est âgé, plus sa probabilité d'appartenir à la première classe est élevée (cf. tableau 6). Ainsi, on peut voir dans les différences entre les deux emplois du temps un effet d'âge, mais aussi de génération. En effet, les tâches ménagères sont moins présentes dans la soirée des plus jeunes ( 25 minutes contre 53 pour les plus âgées) et, si les femmes sont moins nombreuses à travailler à temps partiel, elles vivent aussi un peu moins souvent en couple.

Les prises alimentaires des individus $\mathrm{du}$ deuxième groupe se concentrent sur le début de la soirée. Mais après 20h00, leur part devient inexistante et laisse place à une multitude d'activités. La seconde moitié de la période est consacrée aux loisirs, de la télévision à la lecture en

Tableau 4

Les caractéristiques sociodémographiques des individus des dix classes de 1998

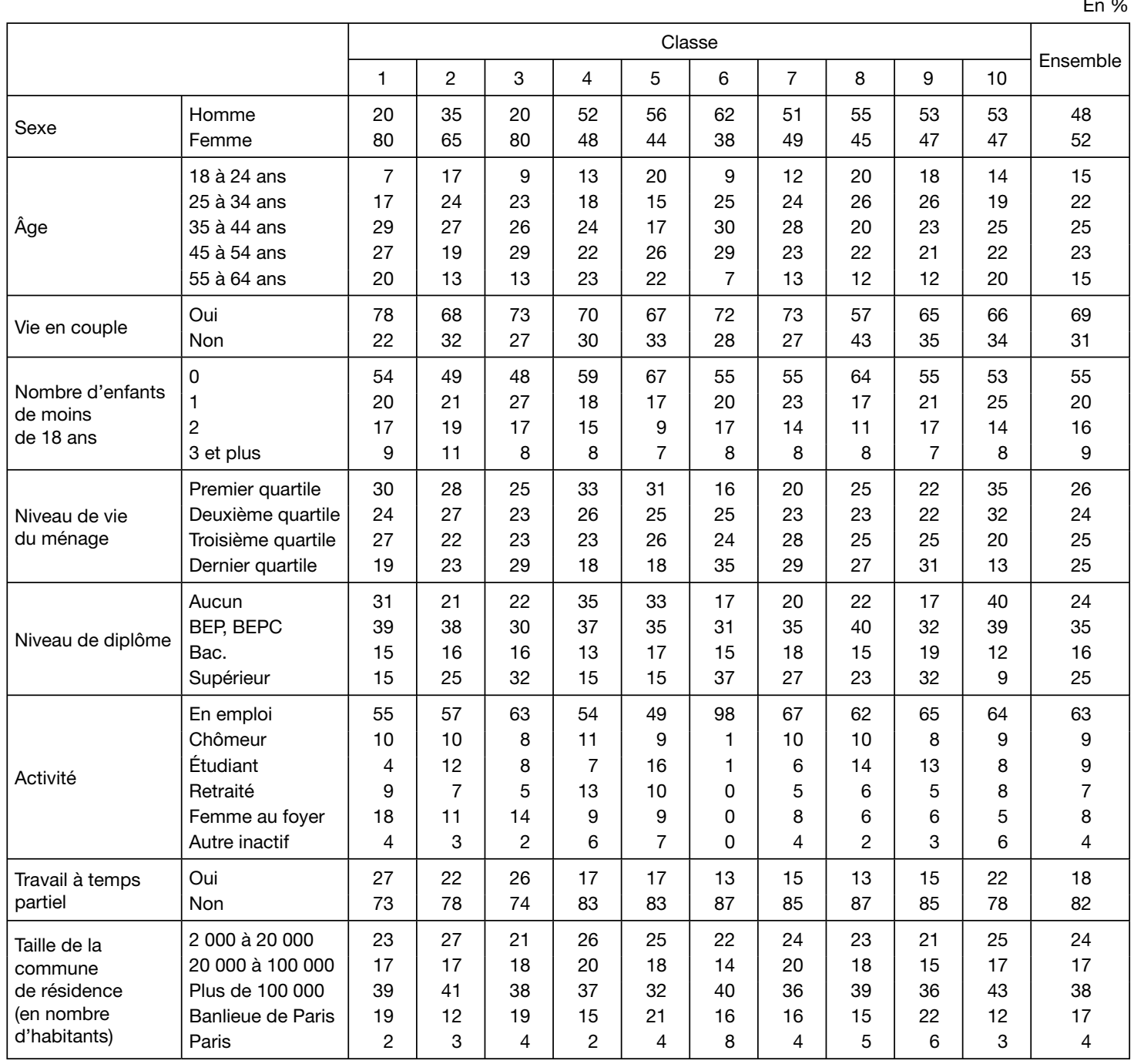

Lecture : 20,3\% des individus contenus dans la première classe sont des hommes. Source : enquête Emploi du temps 1998-1999, Insee. 
passant par la musique, mais aussi tout ce qui a trait à la famille et à la maison (soins et éducation des enfants, etc.). Le travail domestique est important dans les intervalles qui suivent directement le repas, mais s'efface après $21 \mathrm{~h} 00$. Ainsi les activités sont plus diversifiées que dans le groupe précédent où la seconde partie de soirée était dominée par la télévision. La diversité des activités réalisées après le repas semble donc se réduire avec l'augmentation de l'âge.

Le cas de la troisième soirée-type est particulier. Il correspond plus que les deux autres à des femmes actives (et là encore les employées et le personnel de service sont surreprésentés), même si les femmes au foyer y sont encore importantes.

Tableau 5

Les professions et catégories socio-professionnelles sur-représentées par groupe en 1998 (1)

\begin{tabular}{|c|l|}
\hline Classe & \multicolumn{1}{|c|}{ Intitulé (1) } \\
\hline $\mathbf{1}$ & Employés administratifs d'entreprises, Personnels des services directs aux particuliers \\
$\mathbf{2}$ & - \\
$\mathbf{3}$ & Employés administratifs d'entreprises, Personnels des services directs aux particuliers \\
$\mathbf{4}$ & Ouvriers non qualifiés de type industriel \\
$\mathbf{5}$ & - \\
$\mathbf{6}$ & Artisans, Commerçants, Chefs d'entreprise, Professions libérales, Professeurs et professions scientifiques \\
$\mathbf{7}$ & - \\
$\mathbf{8}$ & - \\
$\mathbf{9}$ & Cadres administratifs et commerciaux, Ingénieurs et cadres techniques \\
$\mathbf{1 0}$ & Chauffeurs, Ouvriers non qualifiés (de type industriel et de type artisanal) \\
\hline \multicolumn{2}{|c|}{ (1) Nomenclature à 2 chiffres des professions et catégories socioprofessionnelles (PCS) de l'Insee. } \\
\hline
\end{tabular}

Lecture : les employés administratifs d'entreprises et les personnels des services directs aux particuliers sont des catégories surreprésentées dans la première classe. Sont considérées comme surreprésentées dans une classe les professions dont les contributions au Chi2 total (1 220) sont supérieures à 15.

Source : enquête Emploi du temps 1998-1999, Insee.

Tableau 6

Probabilité d'appartenir à une classe en 1998 (odds ratio)

\begin{tabular}{|c|c|c|c|c|c|c|c|c|c|c|c|}
\hline \multirow{2}{*}{ Variable } & \multirow{2}{*}{ Modalité } & \multicolumn{10}{|c|}{ Classe } \\
\hline & & 1 & 2 & 3 & 4 & 5 & 6 & 7 & 8 & 9 & 10 \\
\hline Genre (vs. homme) & Femme & 3,9 & 1,8 & 4,0 & 0,8 & 0,7 & 0,5 & ns. & 0,7 & 0,7 & 0,7 \\
\hline Age (vs. 25-34) & $\begin{array}{l}18 \text { à } 24 \text { ans } \\
35 \text { à } 44 \text { ans } \\
45 \text { à } 54 \text { ans } \\
55 \text { à } 64 \text { ans }\end{array}$ & $\begin{array}{l}0,6 \\
1,5 \\
1,6 \\
1,8\end{array}$ & & $\begin{array}{l}0,6 \\
1,4\end{array}$ & $\begin{array}{l}\text { ns. } \\
1,2 \\
\text { ns. } \\
1,9\end{array}$ & $\begin{array}{l}2,0 \\
\text { ns. } \\
1,6 \\
1,7\end{array}$ & $\begin{array}{l}0,6 \\
0,3\end{array}$ & 0,7 & 0,5 & $\begin{array}{l}0,8 \\
0,8 \\
0,7\end{array}$ & \\
\hline Vit en couple (vs. non) & Oui & 1,4 & & & & & & & 0,7 & & \\
\hline Nombre d'enfant (vs. 2) & $\begin{array}{l}0 \\
1 \\
3\end{array}$ & & 0,8 & 0,8 & & 1,8 & & $\begin{array}{c}1,4 \\
.\end{array}$ & 1,6 & 0,8 & \\
\hline Niveau de vie (vs. $2^{e}$ quartile) & $\begin{array}{l}1^{\mathrm{er}} \text { quartile }(-) \\
3^{\mathrm{e}} \text { quartile } \\
4^{\mathrm{e}} \text { quartile }(+)\end{array}$ & & & & $\begin{array}{l}1,3 \\
0,8\end{array}$ & 0,7 & 0,6 & $\cdot$ & & $\begin{array}{l}1,2 \\
1,3\end{array}$ & $\begin{array}{l}0,7 \\
0,6\end{array}$ \\
\hline $\begin{array}{l}\text { Niveau de diplôme } \\
\text { (vs. BEP-BEPC) }\end{array}$ & $\begin{array}{l}\text { Aucun } \\
\text { Bac } \\
\text { Supérieur }\end{array}$ & 0,6 & 0,8 & 1,4 & $\begin{array}{l}1,2 \\
0,8 \\
0,6\end{array}$ & 0,7 & 1,4 & & $\begin{array}{l}0,7 \\
0,7\end{array}$ & $\begin{array}{l}1,3 \\
1,4\end{array}$ & $\begin{array}{l}1,4 \\
0,7 \\
0,4\end{array}$ \\
\hline $\begin{array}{l}\text { Taille de la commune } \\
\text { (vs. } 2000 \text { à } 20000 \text { habitants) }\end{array}$ & $\begin{array}{l}20000 \text { à } 100000 \text { habitants } \\
\text { Plus de } 100000 \text { habitants } \\
\text { Banlieue de Paris } \\
\text { Paris }\end{array}$ & 1,4 & $\begin{array}{l}0,6 \\
0,6\end{array}$ & & 0,4 & 1,4 & 1,9 & & & $\begin{array}{l}1,4 \\
1,4\end{array}$ & \\
\hline
\end{tabular}

Lecture: les résultats présentés sont issus de dix régressions logistiques réalisées pour chacune des classes. Ainsi, une femme a 3,9 fois plus de chance d'appartenir à la 1ère classe qu'un homme de même classe d'âge, situation conjugale, nombre d'enfant, niveau de vie et de diplôme et taille de commune. Tous les odds ratio indiqués sont significatifs au seuil de $5 \%$, les nombres non portés sont non significatifs.

Source : enquête Emploi du temps 1998-1999, Insee. 
On peut y voir l'effet de la situation familiale qui amène ces femmes à effectuer après leur journée de travail professionnel un travail domestique. Être diplômé du supérieur accroît la probabilité de se trouver dans ce groupe (cf. tableau 6). Les plus de 44 ans, ceux qui vivent en couple et ont un ou deux enfants y sont nombreux.

La troisième classe est ainsi caractérisée par un repas pris globalement entre $20 \mathrm{~h} 00$ et $21 \mathrm{~h} 00$. La période qui précède le repas est massivement consacrée aux tâches ménagères, et en particulier à la cuisine, activités qui resteront importantes pendant toute la soirée. Les loisirs sont également présents, de manière plus minoritaire. À la différence de la première classe, les travaux ménagers se poursuivent souvent après le repas. Le fort taux d'activité et la grande proportion de femmes dans cette classe font de cette soirée-type une sorte de "deuxième journée des femmes actives » (Maurin, 1989). Si l'homme prend en charge davantage de tâches domestiques lorsque son épouse exerce une activité professionnelle (Zarca, 1990) et que la part des tâches qu'ils accomplissent est d'autant plus grande que la femme gagne plus (Ponthieux et Schreiber, 2006), les femmes actives continuent à assumer la majeure partie des tâches domestiques en dehors de leur activité professionnelle, c'est-à-dire principalement le soir.

Ces trois soirées-types féminines ont donc en commun le temps consacré aux tâches ménagères et une organisation de la soirée totalement différente des classes où les hommes sont majoritaires. Le dîner y occupe une place particulière, non seulement par la part des tâches ménagères qui s'y rapportent, qu'elles effectuent encore plus souvent que les hommes, mais aussi par la structure des activités qui l'encadrent.

\section{Des types masculins d'organisation dans lesquels la télévision tient une grande place}

Regarder la télévision apparaît au contraire comme une pratique plus masculine. Ainsi, la soirée des individus de la quatrième classe, dans laquelle les hommes sont légèrement surreprésentés, est marquée par le découpage de la soirée entre deux activités : une première période est consacrée au repas et s'étend de 19 h00 à 20 h00, plus tôt que les autres groupes. On y observe une forte synchronisation des dîners (jusqu'à $58 \%$ des individus à $19 \mathrm{~h} 30$, cf. annexe 3 ). La seconde période de $20 \mathrm{~h} 00$ à $21 \mathrm{~h} 30$, et sans doute au-delà, est généralement consacrée à la télévision. Cette activité augmente brutalement à $20 \mathrm{~h} 00$, heure où elle passe brutalement de 26 à $68 \%$ pour atteindre $90 \%$ à $20 \mathrm{~h} 50$. Ces individus arrêtent donc leur repas à 20h00, heure du début du journal télévisé et poursuivent cette activité de loisir avec l'émission de première partie de soirée.

Les individus de ce groupe sont sensiblement plus âgés: un individu de plus de 55 ans a deux fois plus de chances de se trouver dans ce groupe qu'un individu qui a entre 25 et 34 ans (cf. tableau 6). Les moins diplômés y sont également surreprésentés (cf. tableau 4). On y trouve également beaucoup de retraités et de chômeurs, mais aussi d'ouvriers non qualifiés. Ce sont globalement des membres de ménage de faible niveau de vie, des individus qui habitent dans une ville petite ou moyenne et qui n'ont majoritairement pas d'enfant. Ce comportement sédentaire, puisque l'activité télévision a lieu presque toujours au domicile, correspond à un habitat où l'offre de loisir est peu élevée ou trop chère. La télévision apparaît ici comme un loisir bon marché et accessible.

Cette pratique est à rapprocher de celle des individus du cinquième groupe, à la différence près que le repas a lieu plus tard et que la télévision est présente avant. Les temps moyens consacrés à la télévision sont très proches et totalisent près d'une heure et demie en moyenne. La télévision est ainsi l'activité largement majoritaire de $19 \mathrm{~h} 00$ à $20 \mathrm{~h} 00$ (98\% des individus à 19h30). Le repas est pris dans la seconde partie de la période étudiée $(20 \mathrm{~h} 00-21 \mathrm{~h} 00)$ de manière concentrée (jusqu'à $76 \%$ des individus à 20h20), puis regarder la télévision redevient à nouveau majoritaire. Les autres activités sont présentes de manière anecdotique, à l'exception des tâches ménagères qui constituent une transition entre les deux périodes. Le repas constitue une rupture entre deux épisodes de télévision qui est peut-être moins décisive qu'il n'y paraît. En effet, il est probable que la télévision n'est pas éteinte, mais qu'elle devient une activité secondaire pendant le temps du dîner.

Ces individus sont majoritairement des hommes et les étudiants, les retraités, les individus peu diplômés et à faible niveau de vie y sont surreprésentés (cf. tableau 4). Avoir moins de 25 ans augmente la probabilité de se trouver dans cette classe, comme dans une moindre mesure le fait d'avoir entre 45 et 64 ans. Pour les membres de cette classe, le dîner s'inscrit vraisemblablement dans un temps consacré à la télévision. $\mathrm{Ce}$ groupe dans lequel les individus inactifs sont 
fortement représentés s'oppose à la classe $6 \mathrm{au}$ fort taux d'activité.

La sixième soirée-type est en effet marquée par l'insertion de l'alimentation dans le temps de travail. La concentration des épisodes alimentaires est faible. À aucun moment plus de $25 \%$ des individus ne mangent en même temps (cf. annexe 3). Le temps consacré à l'alimentation est le plus faible en moyenne de toutes les classes (26 minutes). Par contre, ce groupe se singularise par la place prépondérante du travail et des études auxquels se consacrent sur chaque intervalle entre 33 et $82 \%$ de la population de la classe. On observe une place importante des trajets, qui correspondent sans doute à un retour au domicile. La télévision ne fait son apparition qu'en toute fin de période. Le repas semble ici ne pas être un élément structurant de la soirée. Il est contraint par le travail qui détermine finalement l'espace laissé à l'alimentation.

Du fait de la place importante du temps consacrée à des activités professionnelles, il n'est pas étonnant de trouver dans ce groupe $98 \%$ d'actifs occupant un emploi. Ce sont majoritairement des hommes. Ils ont généralement un niveau de diplôme supérieur au baccalauréat et le plus haut quartile de niveau de vie est surreprésenté. Un Parisien a deux fois plus de chances d'appartenir à cette classe qu'un habitant d'une commune de 2000 ou de 20000 habitants. Cette soirée est typique des professions dont les horaires de travail se prolongent en début de soirée. On y trouve donc en particulier des artisans, des commerçants, des chefs d'entreprises et des professions libérales (cf. tableau 4) qui sont toutes des activités professionnelles où les horaires de travail peuvent se prolonger en soirée. Les enseignants se retrouvent aussi dans ce groupe. Leurs horaires de travail sont également extensifs et il n'est pas rare que leur soirée soit consacrée à la correction de copies ou à la préparation du cours du lendemain (2). Cette soirée-type correspond bien sûr à un jour de semaine travaillé. L'absence de tout travail domestique peut s'expliquer en partie par leur fort taux de vie en couple (72\%), les tâches domestiques étant alors parfois déchargées sur l'emploi du temps du conjoint.

\section{Le repas du samedi soir est particulier}

Les soirées dont rendent compte les groupes issus de l'appariement optimal ne se répartissent pas également le long des jours de la semaine (cf. tableau 7). Ainsi, la classe 6 ne correspond que très rarement à un emploi du temps dominical ce qui n'est gère surprenant puisqu'elle est marquée par la place importante du travail et que le dimanche est généralement chômé. De même, le samedi soir rassemble une part importante des effectifs des septième et huitième classes. Un tiers des emplois du temps du septième type et un quart du huitième correspondent à un samedi.

La septième classe regroupe des individus pour lesquels l'alimentation occupe la majeure partie

2. Pour une description plus précise de l'emploi du temps des enseignants à partir des mêmes données, on pourra se reporter à l'analyse d'Alain Chenu (2002).

Tableau 7

Les soirées-types ne se répartissent pas uniformément le long des jours de la semaine

\begin{tabular}{|c|c|c|c|c|c|c|c|}
\hline \multirow{2}{*}{ Classe } & \multicolumn{7}{|c|}{ Jour observé } \\
\hline & Lundi & Mardi & Mercredi & Jeudi & Vendredi & Samedi & Dimanche \\
\hline 1 & 12 & 17 & 15 & 16 & 15 & 11 & 14 \\
\hline 2 & 11 & 17 & 16 & 21 & 15 & 10 & 10 \\
\hline 3 & 11 & 14 & 18 & 17 & 15 & 14 & 11 \\
\hline 4 & 11 & 18 & 16 & 17 & 13 & 11 & 14 \\
\hline 5 & 11 & 14 & 16 & 13 & 13 & 16 & 17 \\
\hline 6 & 13 & 19 & 18 & 21 & 15 & 8 & 6 \\
\hline 7 & 5 & 11 & 7 & 11 & 17 & 32 & 17 \\
\hline 8 & 8 & 10 & 11 & 16 & 14 & 25 & 16 \\
\hline 9 & 9 & 16 & 17 & 18 & 16 & 13 & 11 \\
\hline 10 & 12 & 16 & 14 & 16 & 17 & 10 & 15 \\
\hline Ensemble & 10 & 16 & 16 & 17 & 15 & 14 & 12 \\
\hline
\end{tabular}

Lecture : 11,8 \% des séquences contenues dans la première classe ont été inscrites un lundi soir. Source : enquête Emploi du temps 1998-1999, Insee. 
de la période considérée. De $19 \mathrm{~h} 00$ à $21 \mathrm{~h} 00$, sur chaque plage de dix minutes, au moins $50 \%$ des individus de ce groupe effectuent une prise alimentaire. À $20 \mathrm{~h} 10$ et à $20 \mathrm{~h} 20$, on a même un pic pendant lequel $98 \%$ des individus de ce groupe mangent. Les autres activités restent peu importantes. Seule la télévision fait une percée après $20 \mathrm{~h} 30$ (cf. annexe 3). Cette soirée-type contient en particulier les repas dont la durée est plus longue que la durée moyenne. On y trouve donc l'ensemble des repas à caractère plus exceptionnel, comme les invitations de parents ou d'amis.

Les personnes ayant un niveau de vie élevé sont surreprésentées dans ce groupe, ainsi que les individus en couple et les actifs. La moyenne élevée (113 minutes) du temps alimentaire sur la période $18 \mathrm{~h} 50$ à $21 \mathrm{~h} 30$ des individus appartenant à cette classe laisse penser que l'alimentation s'inscrit ici dans une pratique de loisir ou de sociabilité, notamment avec des membres extérieurs au ménage.

La classe 8 fait quant à elle apparaître un type de repas très particulier: celui qui correspond à une pratique de sortie. En effet, la catégorie « rencontres, spectacles et promenades », presque absente pour les autres groupes, apparait ici comme l'activité dominante (cf. annexe 3). À $19 \mathrm{~h} 20,66 \%$ des individus de cette classe réalisent une activité de ce type. Il s'agit de pratiques qui impliquent une mobilité de l'individu, car elles sont généralement réalisées à l'extérieur du foyer d'où l'importance des trajets. La télévision apparaît timidement après $21 \mathrm{~h} 00$, heure du retour à la maison pour une partie de ces individus. Le repas n'occupe qu'une place diffuse. Il est étalé sur toute la période et ne connaît qu'une faible concentration autour de $20 \mathrm{~h} 30$.

Mais contrairement aux individus de la septième classe, la population de la huitième est très jeune et les étudiants y sont fortement surreprésentés. Ce sont plutôt des célibataires et ils sont majoritairement sans enfant (64\%). Mais cette huitième soirée-type, caractérisée par la place des sorties, regroupe des cas très différents. Le repas qui précède un spectacle peut être très court. Celui qui accompagne une rencontre peut durer beaucoup plus longtemps. La moyenne est toutefois assez faible (29 minutes).

Cette soirée-type, comme la classe précédente, correspond majoritairement au samedi soir, veille du dimanche généralement chômé. On observe ici une particularité du dîner du samedi, jour pour lequel le dîner dure pour l'ensemble de la population en moyenne vingt minutes de plus qu'un jour de semaine. La classe 7 rend compte de cet étalement du repas, pris au domicile, le samedi soir. La classe 8 saisit la conduite des plus jeunes et des célibataires en particulier pour lesquels le repas est plus souvent pris à l'extérieur. Dans ce second cas, le dîner peut être très court. Nous percevons ici deux modes de vie correspondant à deux places différentes dans le cycle de vie et aux différences de statut conjugal.

\section{L'insertion du repas dans la soirée dépend aussi de l'âge}

La culture de sortie reste donc un trait caractéristique des jeunes qui influe sur l'organisation de l'emploi du temps et sur la place du repas. Bien sûr, ces pratiques de rencontres, sorties au spectacle ou promenades ne sont pas seulement le fait des plus jeunes et des niveaux de vie et de diplôme élevés apparaissent également déterminants quel que soit l'âge. On retrouve d'ailleurs une conclusion de Chenu et Herpin (2002) qui soulignent que "les spectacles et sorties, la participation à la vie associative, la lecture, la pratique des jeux ou de la musique sont plutôt l'apanage des plus diplômés. Les téléspectateurs sont encore plus souvent que par le passé les titulaires du certificat d'étude et les sans diplôme ». Notre typologie rend bien compte de cette différenciation des pratiques de loisirs. Les individus des classes 4 et 5 , pour lesquels la télévision occupe le plus de place dans la période étudiée, sont majoritairement très peu diplômés alors que les individus de la classe 8 ou même de la 9 le sont davantage.

Le mode de vie des plus jeunes ne se distingue pas seulement par cette culture de sortie. La tenue du dîner de manière plus tardive, en seconde partie de période, semble être une caractéristique des individus les plus jeunes, tout comme des plus aisés. En effet, parmi les individus de la neuvième classe, caractérisée par le repas en seconde partie de période, on trouve beaucoup d'étudiants et de personnes entre 18 et 24 ans qui habitent Paris ou sa banlieue, mais on y trouve aussi les cadres et ingénieurs. Dans l'ensemble, ils sont plus souvent diplômés du supérieur et célibataires.

Le repas est concentré entre $20 \mathrm{~h} 00$ et $21 \mathrm{~h} 00$ pour les individus du neuvième groupe, mais il reste important tout au long de la période. Les autres activités, très diverses à $19 \mathrm{~h} 00$, subissent un léger fléchissement à partir de $20 \mathrm{~h} 00$. Une heure plus tard, la structure des activités est toute autre : la télévision occupe une place très 
importante. Le travail, encore présent à 19h00, devient presque inexistant. Le repas constitue ici une sorte de césure dans l'emploi du temps de ce groupe d'individus, marquant une séparation entre une fin de journée où les activités liées à la maison ou la famille occupent une grande place et où les trajets sont encore fortement présents et une soirée plutôt consacrée aux loisirs proprement dits et à la télévision en particulier. Le dîner clôt la journée de travail et ouvre une période consacrée à la détente. Par ailleurs on retrouve l'influence de l'âge sur la diversité des activités : les activités des groupes où les individus sont plus jeunes ou plus aisés apparaissent plus diversifiées que celles des plus âgés et des plus modestes. Mais il ne faut pas oublier que les activités déclarées par les enquêtés sont regroupées dans une nomenclature en 25 postes (cf. annexe 1). Ce regroupement n'est pas neutre et la nomenclature d'activités retenue est peut-être plus propice à la description des activités des plus jeunes et des plus aisés.

Quoi qu'il en soit, cette organisation de la seconde partie de la période étudiée contraste ainsi avec celle d'une partie des plus âgés, qui la consacrent au sommeil. Ainsi la dixième classe regroupe la plupart des personnes qui se couchent avant $21 \mathrm{~h} 30$. L'activité sommeil, quasi absente des emplois du temps des autres groupes, domine la fin de la période (cf. tableau 2 et annexe 3). Le repas est ainsi concentré en tout début de soirée. Sa présence diminue fortement après $20 \mathrm{~h} 00$ pour disparaître totalement après $21 \mathrm{~h} 00$. C'est aussi le cas des autres activités. De $20 \mathrm{~h} 00$ à $21 \mathrm{~h} 00$, une place non négligeable est toutefois dévolue à la télévision. Absente avant et après cet intervalle, la télévision regroupe à $20 \mathrm{~h} 20$ jusqu'à $37 \%$ de la population de cette classe. Mais elle est éteinte avant $21 \mathrm{~h} 00$, heure à laquelle l'activité dominante devient le sommeil pour $95 \%$ d'entre eux et la quasi-totalité de cette classe dans les minutes qui suivent.

Les individus de ce groupe sont marqués par leur âge élevé (cf. tableau 4). Les plus de 55 ans sont surreprésentés. Les individus célibataires et à faible niveau de vie aussi. Ils sont plus souvent peu diplômés et vivent dans des villes petites et moyennes. Les contraintes physiques (fatigue, etc.) et sociales (pas de loisirs adaptés, etc.) influent sur la soirée des plus âgés. Toutefois, il ne s'agit pas du mode de vie de l'ensemble des personnes âgées, surreprésentées aussi dans d'autres classes (cf. tableau 4). En outre, cette pratique n'est pas réservée aux plus âgés et concerne aussi les individus actifs qui, de par leur profession, peuvent avoir une contrainte horaire le lendemain matin de bonne heure comme les chauffeurs et certains ouvriers non qualifiés et se lèvent peut-être plus tôt que les autres (cf. tableau 5).

Au sein de la multitude des emplois du temps de la soirée, il est ainsi possible de mettre en exergue des pratiques propres à certains groupes sociaux. À chacun de ces comportements correspondent des figures de l'insertion du repas au sein des autres activités. L'heure à laquelle le dîner a lieu dépend directement des activités dans lequel il s'inscrit. S'il y avait déplacement ou disparition des repas traditionnels, la cause de cette modification devrait donc aussi être cherchée dans la modification des activités qui entourent le repas et de leur contexte, comme par exemple le recul de l'heure à laquelle commence le film de début de soirée à la télévision.

La télévision tient une place déterminante dans presque toutes les classes et semble influencer directement la durée des autres activités de par ses horaires fixes. Que l'on cuisine, que l'on mange devant sa télévision ou que l'on regarde tous les jours le journal télévisé ou le programme télévisuel de début de soirée après son repas, ces horaires imposés par les programmes télévisés modèlent l'emploi du temps et contribuent au synchronisme alimentaire français. Volatier (1999) relève également le rôle de référence temporelle joué par le journal de 20 heures, souvent regardé à table, pour expliquer que près des trois quarts des Français déclarent manger tous les soirs à la même heure. Mais il y voit également la conséquence de la disponibilité de tous les membres de la famille à un même moment puisque $84 \%$ des familles ou des couples déclarent manger en même temps (Volatier, 1999). Néanmoins, rien dans notre analyse ne permet de certifier que les membres d'un même ménage dînent ensemble puisque nous ne disposons pas des emplois du temps des enfants. Toutefois, pour les mères de famille, l'alimentation des enfants et en particulier le fait qu'ils mangent à heures régulières introduit une contrainte qui pèse sans doute fortement sur les horaires des repas (Marenco, 1992).

Les classes issues de l'appariement des emplois du temps se répartissent en trois groupes selon l'heure du dîner : avant 20h00, après $20 \mathrm{~h} 00$ ou indistinctement tout le long de la période étudiée. La place de ce repas est segmentée socialement. Les soirées-types dans lesquelles le dîner est pris en première moitié de période correspondent plutôt à des individus à faible niveau de vie ou peu diplômés, deux dimensions qui vont 
souvent ensemble. À l'inverse, les soirées-types où le dîner a lieu en seconde moitié de période sont plutôt le fait d'individus à revenu élevé et possédant au moins le baccalauréat. Mais il ne s'agit que de tendances et vivre en couple avec des enfants pousse également plutôt à prendre son repas en première partie de soirée, sans doute à cause de l'heure du coucher de ces derniers, alors que le fait d'être célibataire recule au contraire l'heure du repas. Le pic des dîners cache donc des pratiques différentes, segmentées socialement et spatialement.

Si les modes de vie ne se résument pas à leur seule temporalité, l'emploi du temps individuel en est néanmoins un bon indicateur. Cette analyse a ainsi permis de mettre en lumière différentes pratiques qui constituent autant d'expériences partagées quotidiennement en termes d'horaires par des individus qui possèdent par ailleurs des caractéristiques sociales communes, que ce soit l'âge, le sexe ou encore l'activité professionnelle. Autour de l'organisation de la séquence du dîner sont apparues différentes structures organisationnelles propres à certains groupes sociaux. Plus précisément, on pourrait être tenté de voir derrière tel ou tel type de soirée un habitus, une certaine manière d'organiser sa prise alimentaire en regard d'autres activités, responsable au moins en partie du synchronisme alimentaire observé. Comme ce que l'on mange varie d'un milieu à l'autre (Bourdieu, 1979; Chauvel, 1999 ; Grignon et Grignon, 1999 ; Régnier et al., 2006), le cadre du dîner est différent selon les groupes sociaux. Si l'on prend en compte la dimension d'échange et de sociabilité alimentaire (Larmet, 2002), la synchronisation des repas est un enjeu essentiel dans la cohésion interne d'un groupe et à plus petite échelle d'un couple (Sobal et al., 2002). Le dîner est non seulement un lieu de sociabilité, mais aussi un lieu de socialisation (Blum-Kulka, 1997) pendant lequel les parents transmettent à leurs enfants un certain nombre de valeurs et de règles. Le repas pris en famille joue le rôle d'un « rempart contre la dislocation de la famille, menacée de toutes parts par la vie moderne » (Marenco, 1992) et la concentration française des repas autour des mêmes horaires peut ainsi être aussi lue comme la conséquence de ces comportements familiaux.

\section{Une forte stabilité des pratiques entre 1986 et 1998}

La moitié des classes issues de l'appariement des données de l'enquête Emploi du temps de
1986 ont un effectif très proche des groupes de 1998 dont elles se rapprochent (cf. annexe 2). Les individus appartenant aux première et dixième classes étaient toutefois beaucoup plus nombreux en 1986. La neuvième classe rassemble quant à elle beaucoup plus d'individus en 1998 et la place centrale en 1986 des activités domestiques et familiales disparaît parmi une multitude d'autres activités (cf. annexe 3 ) : ce comportement qui se distinguait en 1986 parmi les dix types se trouve associé en 1998 à d'autres comportements dans une classe plus grande.

Si certaines séquences moyennes sont identiques en 1986 et en 1998, ce sont surtout les graphiques d'activités cumulés qui frappent par leur ressemblance (cf. annexe 3). Les similarités entre les graphiques sont si importantes qu'on pourrait oublier qu'il s'agit de deux études transversales et non longitudinales. Les enquêtes reposent sur deux échantillons totalement différents et, pourtant, les résultats sont très proches, observation qui plaide en faveur d'une assez forte stabilité des pratiques observées.

Ainsi, la quatrième classe de 1986 a une séquence moyenne identique et un graphique similaire à celle de 1998. C'est aussi le cas de la septième classe, même si le pic des repas a lieu un peu plus tôt en 1986 et de la dixième classe avec une avancée notable de l'heure du coucher. Les similitudes sont également très grandes pour les troisième, sixième, et neuvième groupes. Et on peut également rapprocher les première et deuxième classes de 1998 de celle de 1986, même si les séquences moyennes divergent légèrement (3).

Toutefois, on ne retrouve parfaitement en 1986 ni la cinquième ni la huitième classes de 1998 , caractérisées réciproquement par la place de la télévision et de celle des sorties. Ces comportements sont dissous à l'intérieur des autres classes. L'apparition plus nette de la soirée-type consacrée aux sorties dans la typologie de 1998 peut être lue comme le signe d'une dynamique au sein de nos emplois du temps. L'accroissement de la tendance de sorties pendant cette partie de la soirée est une des évolutions notables entre les deux enquêtes. L'accroissement du nombre de repas pris à l'extérieur du domicile est en

3. En effet, le fait que la séquence au centre de la classe soit différente entre 1986 et 1998 n'a rien de surprenant. Ainsi le fait que les éléments " Enfants » ou "Bricolage/Jardin » n'apparaissent plus dans les séquences-moyennes de 1986 ne peut être interprété seul, puisque rien n'indique que ces activités ne soient pas dans les autres séquences de la classe. Pour cela, il faut observer le graphique des effectifs cumulés. 
effet une tendance relevée également par exemple par Fischler (1990) ou par Poulain (2002), même si les repas effectués au domicile constituent encore l'essentiel des dîners (Michaud et $a l ., 2004)$ et si ce développement a lieu lentement (Grignon et Grignon, 1999) et ne semble concerner principalement que le week-end (Volatier, 1999).

De même, l'apparition en 1998 d'une classe dans laquelle la télévision tient une grande place signale un renforcement de cette pratique et de son lien avec le "modèle » alimentaire en France. L'heure de fin du dîner semble fortement corrélée aux horaires des programmes télévisuels et en particulier du journal télévisé, et ce pour presque tous les groupes. Entre les deux enquêtes, la période où la télévision est la plus regardée après le repas semble s'être décalée. Cette activité débute en effet plus souvent à 21 h00 qu'à $20 \mathrm{~h} 30$ en 1998, évolution parallèle au recul de l'heure du début des programmes télévisuels de début de soirée dans la même période (cf. annexe 3 ).

Au-delà de cette similitude dans les séquences inscrites par les individus des deux enquêtes, on retrouve des caractéristiques sociodémographiques similaires pour les individus des classes de 1986 (cf. annexe 2). Ainsi, par exemple, comme pour 1998, les trois premières classes sont essentiellement féminines, même si les hommes sont un peu plus nombreux à avoir une soirée du premier type. Les hommes restent majoritaires dans les soirées dans lesquelles la télévision occupe une grande place. Avoir un faible niveau d'éducation augmente la probabilité d'avoir une soirée dans laquelle le sommeil occupe une grande place et avoir un niveau de vie élevé la diminue.

Des différences apparaissent également, comme le fait que la neuvième classe soit en 1986 une classe très féminine, dont les individus consacrent une place plus importante aux activités domestiques et familiales (cf. annexe 3). Mais le fait d'être diplômé du supérieur ou parisien augmente toujours la probabilité d'appartenir à ce groupe. Par ailleurs, certaines modalités qui influaient sur la probabilité d'appartenir à une classe ne sont plus significatives, tandis que d'autres le deviennent. C'est le cas par exemple des classes d'âges qui ne sont pas significatives pour la probabilité d'appartenir à la première classe. Toutefois, ces différences apparaissent assez mineures par rapport aux similitudes entres les résultats de 1986 et 1998.

Du point de vue du dîner, la comparaison des deux enquêtes fait apparaître une assez forte stabilité des plages alimentaires. Que ce soit en 1986 ou en 1998, le dîner est toujours présent et occupe une place de choix dans la soirée des Français. Pour les deux années, nous avons observé des épisodes alimentaires contigus, preuve de la persistance du repas du soir, et non une multiplication de petites tranches horaires dévolues à l'alimentation. Il n'apparaît pas de véritable déstructuration du dîner. Comme le notent Aymard et al. (1993), "si l'usage se fixe et se maintient, c'est précisément parce qu'il n'est pas une structure, et qu'il suffit, pour le respecter, de le suivre dans la mesure du possible, et parfois d'assez loin ». L'appariement optimal des emplois du temps a justement permis de regrouper ces déclinaisons d'une même pratique. Les dix groupes, qui correspondent à dix environnements différents du dîner partagés par des individus dont les caractéristiques sociales sont souvent assez proches, constituent une tentative de description du contexte et des logiques propres au repas du soir.

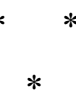

Si cette étude ne permet pas de se prononcer précisément sur la multiplication des prises alimentaires « hors repas », il apparaît clairement que si cette multiplication existe, elle n'affecte pas directement le dîner, qui constitue encore un repas « réglé », une étape obligée dans l'emploi du temps quotidien des Français, conclusion similaire à celle qu'obtient Metsdag (2005) pour les Flamands, d'où la forte synchronisation des prises alimentaires durant cet intervalle de temps en France. L'étude des emplois du temps nous a permis d'approcher les pratiques qui se déroulent pendant le « pic des dîners ». Non seulement on observe ce pic en 1986 et en 1998, mais il n'y a pas de bouleversement apparent des comportements le composant. Nous retrouvons pour presque toutes nos classes de 1998 une soirée-type déjà construite en 1986, qui traduisent ainsi une tendance solide du comportement des Français. La disparition et la déstructuration du repas du soir ne semblent pas être encore d'actualité. 


\section{BIBLIOGRAPHIE}

Abbott A. (1995), «Sequence Analysis: New Methods for Old Ideas », Annual Review of Sociology, vol. 21, pp. 93-113.

Abbott A. et Hrycak A. (1990), «Measuring Resemblance in Sequence Analysis: An Optimal Matching Analysis of Musicians Careers "), American Journal of Sociology, vol. 96, pp. 144-185.

Aymard M., Grignon C. et Sabban F. (1993), Le temps de manger, Éditions de la Maison des sciences de l'homme, Inra.

Basdevant A., Craplet C. et Guy-Grand B. (1993), «Snacking Patterns in Obese French Women », Appetite, vol. 21, pp. 17-23.

Blum-Kulka S. (1997), Dinner Talk: Cultural Patterns of Sociability and Socialization in Family Discourse, Lawrence Erlbaum Associates, Mahwah.

Brousse C. (1999), «La répartition du travail domestique entre conjoints reste très largement spécialisée et inégale », France, portrait social, pp. 135-151.

Bourdieu P. (1979), La Distinction: critique sociale du jugement, Éditions de Minuit, Paris.

Chenu A. et Herpin N. (2002), "Une pause dans la marche vers la civilisation des loisirs ? », Économie et Statistique, no 352-353, pp. 15-37.

Chenu A. (2002), « Les horaires et l'organisation du temps de travail », Économie et Statistique, $\mathrm{n}^{\mathrm{o}} 352-353$, pp. 151-168.

Chauvel L. (1999), « Du pain et des vacances : la consommation des catégories socioprofessionnelles s'homogénéise-t-elle (encore) ? », Revue française de sociologie, vol. LX, pp. 79-96.

Fischler C. (1979), « Gastro-nomie et gastroanomie : sagesse du corps et crise bioculturelle de l'alimentation moderne », Communications, no 31, pp. 189-210.

Fischler C. (1990), L'homnivore, Éditions Odile Jacob, Paris.

Fischler C. (1996), « Le repas familial vu par les 10-11 ans », Les Cahiers de l'OCHA, nº 6.

Grignon C. (1987), L'alimentation des étudiants, Inra-Cnous, Paris.
Grignon C. (1998), La vie matérielle des étudiants, La Documentation française, Paris.

Grignon C. et Grignon Ch. (1999), « Long-term Trends in Food Consumption: a French Portrait », Food and Foodways, vol. 8, no 3, pp. 151-174.

Herpin N. (1988), « Le repas comme institution », Revue française de sociologie, vol. 39, pp. 503-521.

Larmet G. (2002), « La sociabilité alimentaire s'accroît », Économie et Statistique, no $352-353$, pp. 191-211.

Lesnard L.et de Saint Pol T.(2006), « Introduction aux méthodes d'appariement optimal (Optimal Matching Analysis) », Bulletin de méthodologie sociologique, $\mathrm{n}^{\circ} 90$, pp. 5-25.

Lesnard L. (2006), "Optimal Matching and Social Sciences », Document de travail du CREST, $\mathrm{n}^{\mathrm{o}}$ 2006-01.

Marenco C. (1992), Manières de table, modèles

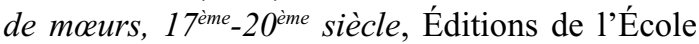
Normale Supérieure de Cachan.

Maurin E. (1989), « Types de pratiques, types de journées et déterminants sociaux de la vie quotidienne », Économie et Statistique, $\mathrm{n}^{\circ} 223$, pp. 25-46.

Mestdag I. (2005), "Disappearance of the Traditional Meal : Temporal, Social and Spatial Destructuration », Appetite, n 45, pp. 62-74.

Michaud C., Baudier F., Guilbert P., Carel D., Le Bihan G., Gautier A. et Delamarie C. (2004), «Les repas des Français : résultats du baromètre santé nutrition », Cahiers Français de Nutrition et de Diététique, vol. 39, n 3, pp. 203-209.

Ponthieux S., Schreiber A. (2006), « Dans les couples de salariés, la répartition du travail domestique reste inégale », Données sociales 2006, pp. 43-51.

Poulain J.-P. (2002), Sociologies de l'alimentation, Presses Universitaires de France, Paris.

Pynson P. (1987), La France à table, Éditions La Découverte, Paris.

Régnier F., Lhuissier A. et Gojard S. (2006), Sociologie de l'alimentation, Éditions $\mathrm{La}$ Découverte, Paris. 
de Saint Pol T. (2005a), «Quand est-ce qu'on mange ? Le temps des repas en France », Terrains et travaux, $\mathrm{n}^{\circ} 9$, pp. 51-72.

de Saint Pol T. (2005b), « Le dîner des Français : étude séquentielle d'un emploi du temps », Document de travail du CREST, $\mathrm{n}^{\circ}$ 2005-19, Insee.

de Saint Pol T. (2006), « Corps et appartenance sociale: le cas de la corpulence en Europe », Données sociales 2006, pp. 649-56.

Sobal J., Bove C. et Rauschenbach B. (2002), «Commensal Careers at Entry into Marriage:
Establishing Commensal Units and Managing Commensal Circles », Sociological Review, ${ }^{\circ}$ 50, pp. 378-97.

Szalai A. (1972), The Use of Time, Mouton, The Hague.

Volatier J.-L. (1999), « Le repas traditionnel se porte encore bien », Consommation et modes de vie, $\mathrm{n}^{\circ} 132$, Crédoc.

Zarca B. (1990), « La division du travail domestique, poids du passé et tensions au sein du couple », Économie et statistique, $\mathrm{n}^{\circ} 228$, pp. 29-40. 
ANNEXE 1

NOMENCLATURE

Tableau A

Correspondance entre les nomenclatures (1)

\begin{tabular}{|c|c|}
\hline Nomenclature en 25 postes & Nomenclature en 9 postes \\
\hline Sommeil & Sommeil \\
\hline Repas & Repas \\
\hline $\begin{array}{l}\text { Travail professionnel } \\
\text { Études }\end{array}$ & Travail / Études \\
\hline $\begin{array}{l}\text { Déplacements domicile-travail } \\
\text { Trajets loisirs ou domicile }\end{array}$ & Trajets \\
\hline Cuisine, linge, ménage & Cuisine / Linge / ménage \\
\hline $\begin{array}{l}\text { Soins et éducation des enfants } \\
\text { Courses } \\
\text { Bricolage, Jardin, Soins animaux } \\
\text { Couture } \\
\text { Travaux domestiques divers } \\
\text { Toilette, Soins personnels }\end{array}$ & Maison et Famille \\
\hline $\begin{array}{l}\text { Religion, Cimetière } \\
\text { Associations } \\
\text { Jeux, Musique } \\
\text { Conversation } \\
\text { Lecture } \\
\text { Radio, Écoute musique } \\
\text { Détente, Pauses }\end{array}$ & $\begin{array}{l}\text { Loisirs } \\
\text { (autres que la télévision et les sorties) }\end{array}$ \\
\hline $\begin{array}{l}\text { Spectacles } \\
\text { Rencontres } \\
\text { Sport } \\
\text { Promenade, Plage, Pêche, Chasse }\end{array}$ & Rencontres / Spectacles / Promenades \\
\hline Télévision & Télévision \\
\hline
\end{tabular}




\section{LES RÉSULTATS DE 1986}

Tableau A

Effectifs des dix classes de 1986 et de 1996

\begin{tabular}{|c|c|c|c|c|}
\hline & \multicolumn{2}{|c|}{ En 1986 } & \multicolumn{2}{c|}{ En 1998 } \\
\hline Classe & Effectif & En \% & Effectif & 5,8 \\
\hline $\mathbf{1}$ & 1010 & 10,1 & 982 & 12 \\
\hline $\mathbf{2}$ & 1347 & 13,5 & 617 & 7,5 \\
\hline $\mathbf{3}$ & 741 & 7,4 & 1713 & 20,8 \\
\hline $\mathbf{4}$ & 1719 & 17,2 & 445 & 5,4 \\
\hline $\mathbf{5}$ & 1152 & 11,5 & 778 & 9,4 \\
\hline $\mathbf{6}$ & 721 & 7,2 & 497 & 6 \\
\hline $\mathbf{7}$ & 405 & 4,1 & 401 & 4,9 \\
\hline $\mathbf{8}$ & 1798 & 18 & 2021 & 24,5 \\
\hline $\mathbf{9}$ & 368 & 3,7 & 303 & 3,7 \\
\hline $\mathbf{1 0}$ & 714 & 7,2 & & \\
\hline
\end{tabular}

Lecture : la classe 1 comprend 1010 séquences, soit 10,1\% de la population totale. Source : enquête Emploi du temps 1985-1986 et 1998-1999, Insee.

Tableau B

Les séquences moyennes de 1986

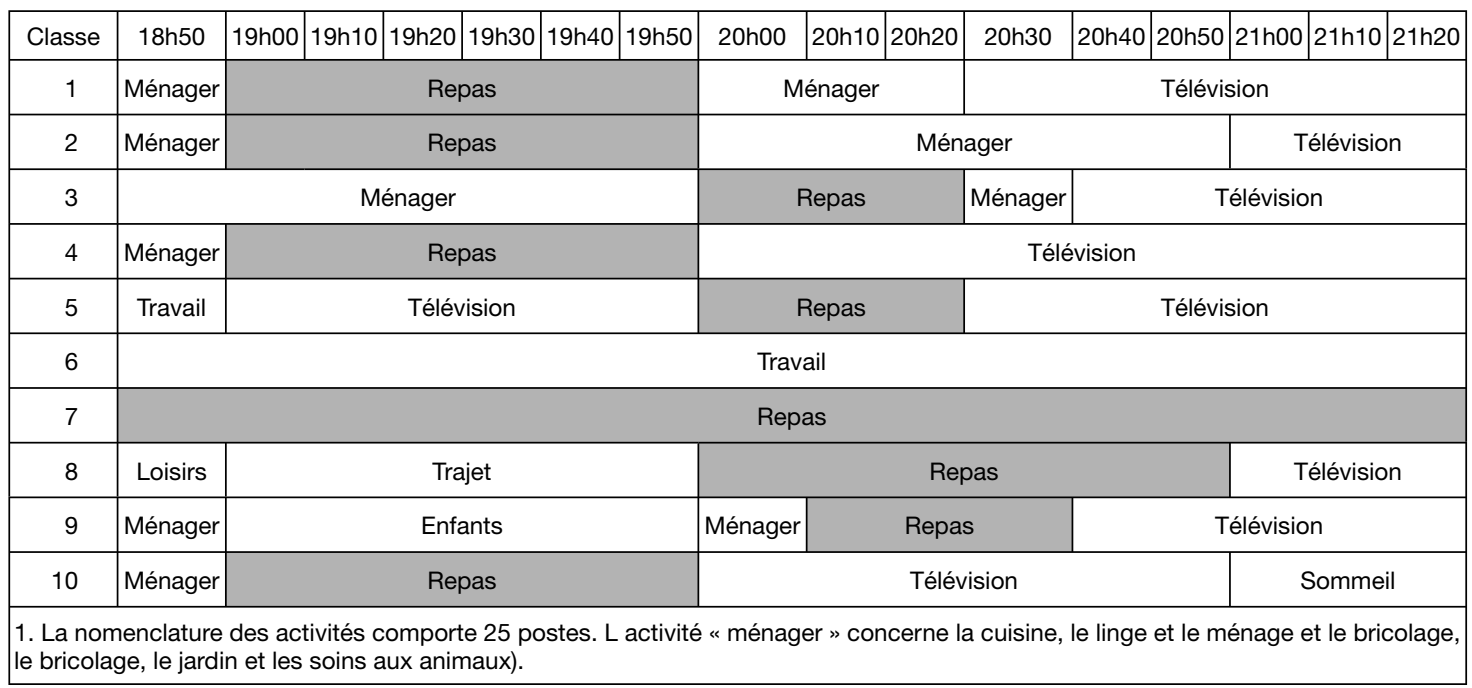

Lecture : à 18h50, l'individu au centre de la première classe a inscrit une activité de type "cuisine-linge-ménage ». Source : enquête Emploi du temps 1998-1999, Insee. 
Tableau C

Les caractéristiques sociodémographiques des individus des dix classes en 1986

\begin{tabular}{|c|c|c|c|c|c|c|c|c|c|c|c|c|}
\hline & & \multicolumn{10}{|c|}{ Classe } & \multirow{2}{*}{ Ensemble } \\
\hline & & 1 & 2 & 3 & 4 & 5 & 6 & 7 & 8 & 9 & 10 & \\
\hline \multirow{2}{*}{ Sexe } & Homme & 35 & 38 & 17 & 57 & 57 & 61 & 47 & 51 & 24 & 48 & 46 \\
\hline & Femme & 65 & 62 & 83 & 43 & 43 & 39 & 53 & 49 & 76 & 52 & 54 \\
\hline \multirow{5}{*}{ Âge } & 18 à 24 ans & 10 & 13 & 7 & 8 & 8 & 8 & 11 & 17 & 11 & 10 & 11 \\
\hline & 25 à 34 ans & 33 & 34 & 27 & 23 & 28 & 28 & 37 & 30 & 62 & 24 & 30 \\
\hline & 35 à 44 ans & 26 & 25 & 27 & 21 & 24 & 30 & 28 & 25 & 21 & 22 & 24 \\
\hline & 45 à 54 ans & 15 & 14 & 21 & 19 & 20 & 21 & 11 & 15 & 4 & 19 & 17 \\
\hline & 55 à 64 ans & 16 & 14 & 18 & 29 & 20 & 13 & 13 & 13 & 2 & 25 & 18 \\
\hline \multirow{2}{*}{ Vie en couple } & Oui & 86 & 76 & 83 & 80 & 84 & 82 & 78 & 71 & 90 & 79 & 80 \\
\hline & Non & 14 & 24 & 17 & 20 & 16 & 18 & 22 & 29 & 10 & 21 & 20 \\
\hline \multirow{4}{*}{$\begin{array}{l}\text { Nombre d'enfants } \\
\text { de moins } \\
\text { de } 18 \text { ans }\end{array}$} & 0 & 41 & 47 & 48 & 57 & 52 & 51 & 48 & 58 & 4 & 56 & 50 \\
\hline & 1 & 24 & 23 & 25 & 19 & 22 & 20 & 26 & 19 & 35 & 18 & 22 \\
\hline & 2 & 25 & 21 & 18 & 16 & 19 & 20 & 20 & 16 & 41 & 16 & 20 \\
\hline & 3 et plus & 10 & 9 & 9 & 8 & 7 & 9 & 6 & 7 & 20 & 10 & 8 \\
\hline \multirow{4}{*}{$\begin{array}{l}\text { Niveau de vie } \\
\text { du ménage }\end{array}$} & Premier quartile & 24 & 26 & 24 & 25 & 26 & 25 & 22 & 25 & 26 & 27 & 25 \\
\hline & Deuxième quartile & 25 & 23 & 25 & 25 & 25 & 25 & 29 & 26 & 26 & 26 & 25 \\
\hline & Troisième quartile & 25 & 27 & 23 & 25 & 22 & 25 & 23 & 24 & 27 & 26 & 25 \\
\hline & Dernier quartile & 26 & 24 & 28 & 25 & 27 & 25 & 26 & 25 & 21 & 21 & 25 \\
\hline \multirow{4}{*}{ Niveau de diplôme } & Aucun & 40 & 32 & 38 & 47 & 37 & 32 & 29 & 28 & 26 & 50 & 37 \\
\hline & BEP, BEPC & 41 & 32 & 35 & 35 & 35 & 36 & 34 & 35 & 38 & 31 & 35 \\
\hline & Bac. & 10 & 16 & 14 & 10 & 12 & 11 & 14 & 15 & 14 & 10 & 13 \\
\hline & Supérieur & 9 & 20 & 13 & 8 & 16 & 21 & 23 & 22 & 22 & 9 & 15 \\
\hline \multirow{6}{*}{ Activité } & En emploi & 59 & 66 & 66 & 57 & 73 & 98 & 75 & 71 & 57 & 63 & 68 \\
\hline & Chômeur & 6 & 7 & 5 & 7 & 5 & 0 & 6 & 5 & 7 & 6 & 6 \\
\hline & Étudiant & 3 & 4 & 1 & 2 & 2 & 1 & 1 & 7 & 1 & 2 & 3 \\
\hline & Retraité & 7 & 5 & 6 & 14 & 6 & 0 & 5 & 5 & 1 & 9 & 7 \\
\hline & Femme au foyer & 21 & 14 & 20 & 15 & 11 & 1 & 10 & 8 & 30 & 15 & 13 \\
\hline & Autre inactif & 4 & 4 & 2 & 5 & 3 & 0 & 3 & 4 & 4 & 5 & 3 \\
\hline \multirow{2}{*}{$\begin{array}{l}\text { Travail à temps } \\
\text { partiel }\end{array}$} & Oui & 13 & 11 & 15 & 6 & 6 & 6 & 9 & 8 & 16 & 10 & 9 \\
\hline & Non & 87 & 89 & 85 & 94 & 94 & 94 & 91 & 92 & 84 & 90 & 91 \\
\hline \multirow{5}{*}{$\begin{array}{l}\text { Taille de la } \\
\text { commune } \\
\text { de résidence } \\
\text { (en nombre } \\
\text { d'habitants) }\end{array}$} & 2000 à 20000 & 22 & 22 & 20 & 26 & 19 & 24 & 18 & 19 & 21 & 26 & 22 \\
\hline & 20000 à 100000 & 20 & 20 & 16 & 19 & 15 & 17 & 15 & 16 & 17 & 16 & 17 \\
\hline & Plus de 100000 & 38 & 37 & 40 & 38 & 40 & 38 & 42 & 38 & 40 & 37 & 38 \\
\hline & Banlieue de Paris & 17 & 15 & 20 & 13 & 21 & 14 & 17 & 19 & 17 & 14 & 17 \\
\hline & Paris & 3 & 6 & 4 & 4 & 5 & 7 & 8 & 8 & 5 & 7 & 6 \\
\hline
\end{tabular}

Lecture : 20,3 \% des individus contenus de la première classe sont des hommes.

Source : enquête Emploi du temps 1986, Insee. 
Tableau D

Probabilité d'appartenir à une classe en 1986 (odds ratio)

\begin{tabular}{|c|c|c|c|c|c|c|c|c|c|c|c|}
\hline \multirow{2}{*}{ Variable } & \multirow{2}{*}{ Modalité } & \multicolumn{10}{|c|}{ Classe } \\
\hline & & 1 & 2 & 3 & 4 & 5 & 6 & 7 & 8 & 9 & 10 \\
\hline Sexe (vs. homme) & Femme & 1,7 & 1,5 & 4,9 & 0,6 & 0,6 & 0,5 & ns. & 0,8 & 2,7 & ns. \\
\hline Age (vs. 25-34) & $\begin{array}{l}18 \text { à } 24 \text { ans } \\
35 \text { à } 44 \text { ans } \\
45 \text { à } 54 \text { ans } \\
55 \text { à } 64 \text { ans }\end{array}$ & $\begin{array}{l}\text { ns. } \\
\text { ns. } \\
\text { ns. } \\
\text { ns. }\end{array}$ & $\begin{array}{l}\text { ns. } \\
\text { ns. } \\
0,8 \\
0,8\end{array}$ & $\begin{array}{r}0,6 \\
1,4 \\
1,5 \\
\text { ns. }\end{array}$ & $\begin{array}{l}\text { ns. } \\
\text { ns. } \\
1,4 \\
2,1\end{array}$ & $\begin{array}{l}\text { ns. } \\
\text { ns. } \\
1,3 \\
\text { ns. }\end{array}$ & $\begin{array}{l}\text { ns. } \\
1,3 \\
\text { ns. } \\
0,7\end{array}$ & $\begin{array}{l}\text { ns. } \\
\text { ns. } \\
0,5 \\
0,6\end{array}$ & $\begin{array}{l}1,4 \\
\text { ns. } \\
0,8 \\
0,6\end{array}$ & $\begin{array}{l}\text { ns. } \\
0,3 \\
0,3 \\
0,4\end{array}$ & $\begin{array}{l}\text { ns. } \\
\text { ns. } \\
\text { ns. } \\
1,5\end{array}$ \\
\hline Vit en couple (vs. non) & Oui & 1,5 & 0,8 & 1,3 & ns. & 1,3 & ns. & ns. & 0,7 & ns. & ns. \\
\hline Nombre d'enfant (vs. 2) & $\begin{array}{l}0 \\
1 \\
3\end{array}$ & $\begin{array}{l}0,7 \\
0,8 \\
\text { ns. }\end{array}$ & $\begin{array}{l}\text { ns. } \\
\text { ns. } \\
\text { ns. }\end{array}$ & $\begin{array}{l}\text { ns. } \\
1,3 \\
\text { ns. }\end{array}$ & $\begin{array}{l}\text { ns. } \\
\text { ns. } \\
\text { ns. }\end{array}$ & $\begin{array}{l}\text { ns. } \\
\text { ns. } \\
0,7\end{array}$ & $\begin{array}{l}\text { ns. } \\
\text { ns. } \\
\text { ns. }\end{array}$ & $\begin{array}{l}\text { ns. } \\
\text { ns. } \\
\text { ns. }\end{array}$ & $\begin{array}{l}1,6 \\
\text { ns. } \\
\text { ns. }\end{array}$ & $\begin{array}{l}0,0 \\
0,7 \\
\text { ns. }\end{array}$ & $\begin{array}{l}\text { ns. } \\
\text { ns. } \\
\text { ns. }\end{array}$ \\
\hline Niveau de vie (vs. $2^{e}$ quartile) & $\begin{array}{l}1^{\mathrm{er}} \text { quartile (-) } \\
3^{\mathrm{e}} \text { quartile } \\
4^{\mathrm{e}} \text { quartile (+) }\end{array}$ & $\begin{array}{l}\text { ns. } \\
\text { ns. } \\
\text { ns. }\end{array}$ & $\begin{array}{l}\text { ns. } \\
1,2 \\
\text { ns. }\end{array}$ & $\begin{array}{l}\text { ns. } \\
\text { ns. } \\
\text { ns. }\end{array}$ & $\begin{array}{l}\text { ns. } \\
\text { ns. } \\
\text { ns. }\end{array}$ & $\begin{array}{l}\text { ns. } \\
\text { ns. } \\
\text { ns. }\end{array}$ & $\begin{array}{l}\text { ns. } \\
\text { ns. } \\
\text { ns. }\end{array}$ & $\begin{array}{l}0,8 \\
\text { ns. } \\
\text { ns. }\end{array}$ & $\begin{array}{l}\text { ns. } \\
\text { ns. } \\
\text { ns. }\end{array}$ & $\begin{array}{l}\text { ns. } \\
\text { ns. } \\
\text { ns. }\end{array}$ & $\begin{array}{l}\text { ns. } \\
\text { ns. } \\
0,8\end{array}$ \\
\hline $\begin{array}{l}\text { Niveau de diplôme (vs. BEP- } \\
B E P C \text { ) }\end{array}$ & $\begin{array}{l}\text { Aucun } \\
\text { Bac } \\
\text { Supérieur }\end{array}$ & $\begin{array}{l}\text { ns. } \\
0,7 \\
0,5\end{array}$ & $\begin{array}{l}\text { ns. } \\
1,5 \\
1,5\end{array}$ & $\begin{array}{l}\text { ns. } \\
\text { ns. } \\
0,8\end{array}$ & $\begin{array}{l}1,2 \\
0,7 \\
0,5\end{array}$ & $\begin{array}{l}\text { ns. } \\
\text { ns. } \\
\text { ns. }\end{array}$ & $\begin{array}{c}\text { ns. } \\
\text { ns. } \\
1,3\end{array}$ & $\begin{array}{l}\text { ns. } \\
\text { ns. } \\
1,4\end{array}$ & $\begin{array}{l}0,8 \\
1,2 \\
1,5\end{array}$ & $\begin{array}{l}0,7 \\
\text { ns. } \\
1,6\end{array}$ & $\begin{array}{l}1,5 \\
\text { ns. } \\
0,6\end{array}$ \\
\hline $\begin{array}{l}\text { Taille de la commune (vs. } \\
2000 \text { à } 20000 \text { habitants) }\end{array}$ & $\begin{array}{l}20000 \text { à } 100000 \text { habitants } \\
\text { Plus de } 100000 \text { habitants } \\
\text { Banlieue de Paris } \\
\text { Paris }\end{array}$ & $\begin{array}{l}\text { ns. } \\
\text { ns. } \\
\text { ns. } \\
\text { ns. }\end{array}$ & $\begin{array}{l}\text { ns. } \\
\text { ns. } \\
0,8 \\
\text { ns. }\end{array}$ & $\begin{array}{l}\text { ns. } \\
\text { ns. } \\
1,4 \\
\text { ns. }\end{array}$ & $\begin{array}{l}\text { ns. } \\
\text { ns. } \\
0,7 \\
0,6\end{array}$ & $\begin{array}{l}\text { ns. } \\
1,2 \\
1,5 \\
\text { ns. }\end{array}$ & $\begin{array}{l}\text { ns. } \\
\text { ns. } \\
0,7 \\
\text { ns. }\end{array}$ & $\begin{array}{l}\text { ns. } \\
\text { ns. } \\
\text { ns. } \\
\text { ns. }\end{array}$ & $\begin{array}{r}\text { ns } \\
\text { ns } \\
1,2 \\
1,4\end{array}$ & $\begin{array}{l}\text { ns. } \\
\text { ns. } \\
\text { ns. } \\
\text { ns. }\end{array}$ & $\begin{array}{l}\text { ns. } \\
\text { ns. } \\
\text { ns. } \\
\text { ns. }\end{array}$ \\
\hline
\end{tabular}

Lecture : les résultats présentés sont issus de dix régressions logistiques réalisées pour chacune des classes. Ainsi, une femme a 1,7 fois plus de chance d'appartenir à la 1ère classe qu'un homme de même classe d'âge, situation conjugale, nombre d'enfant, niveaux de vie et de diplôme et taille de commune. Tous les odds ratio indiqués sont significatifs au seuil de $5 \%$, les nombres non portés sont non significatifs.

Source : enquête Emploi du temps 1986, Insee. 


\section{LES EFFECTIFS CUMULÉS POUR 1986 ET 1998}

Classe 1

Un repas encadré de tâches ménagères

\section{A. En 1998-1999}

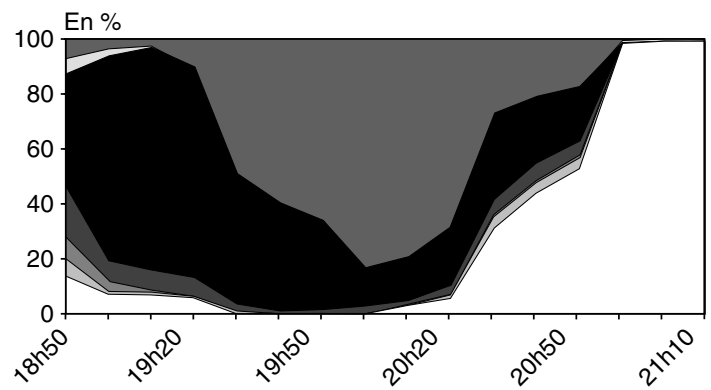

B. En 1985-1986

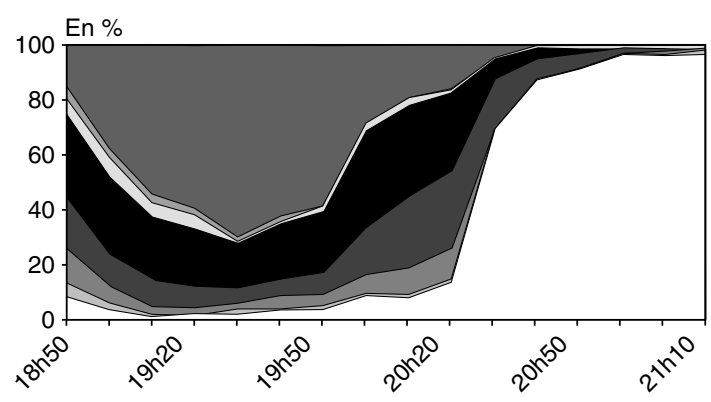

Classe 2

Un repas en première moitié de période, suivi par une multitude d'activités

A. En 1998-1999

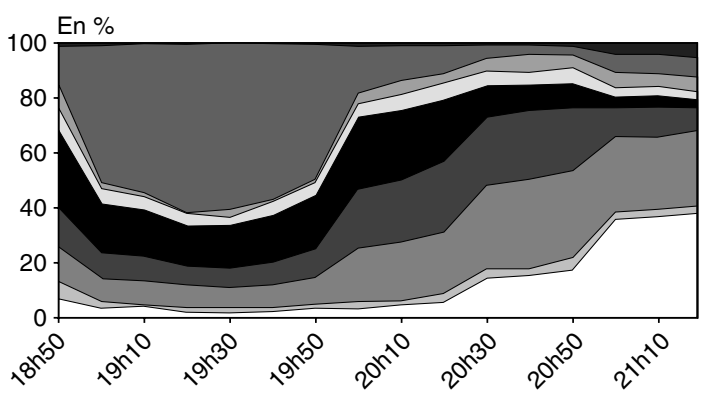

B. En 1985-1986

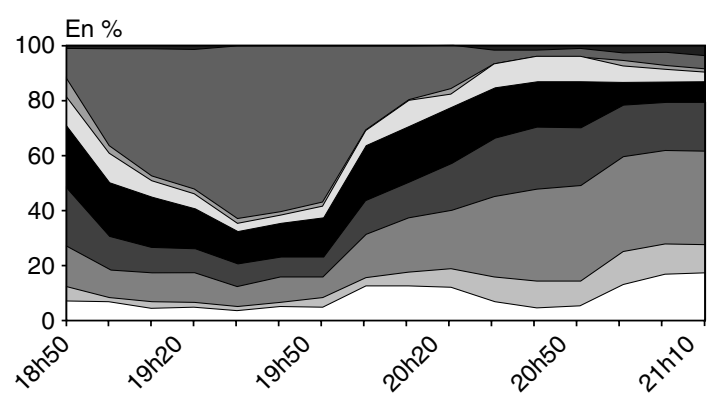

Classe 3

Un repas en seconde moitié de période, encadré de tâches ménagères

A. En 1998-1999

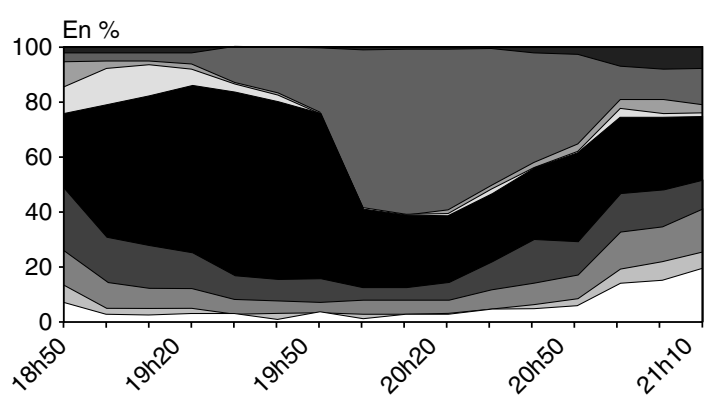

Sommeil

$\square$ Repas

$\square$ Travail / Études

$\square$ Trajets

Cuisine / Linge / Ménage

Maison / Famille

$\square$ Loisirs (autres que Télévision et Sorties)

$\square$ Rencontres / Spectacles / Promenades

$\square$ Télévision
B. En 1985-1986

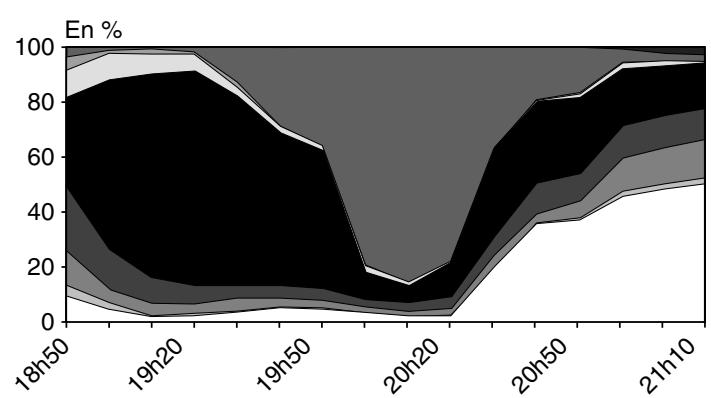

Sommeil

$\square$ Repas

$\square$ Travail / Études

$\square$ Trajets

Cuisine / Linge / Ménage

Maison / Famille

$\square$ Loisirs (autres que Télévision et Sorties)

$\square$ Rencontres / Spectacles / Promenades

$\square$ Télévision 
Classe 4

Une soirée découpée entre le repas et la télévision

A. En 1998-1999

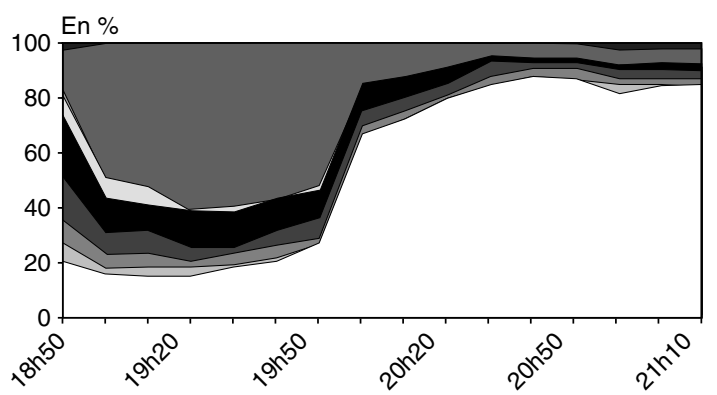

Classe 5

Une soirée entièrement consacrée à la télévision

\section{A. En 1998-1999}

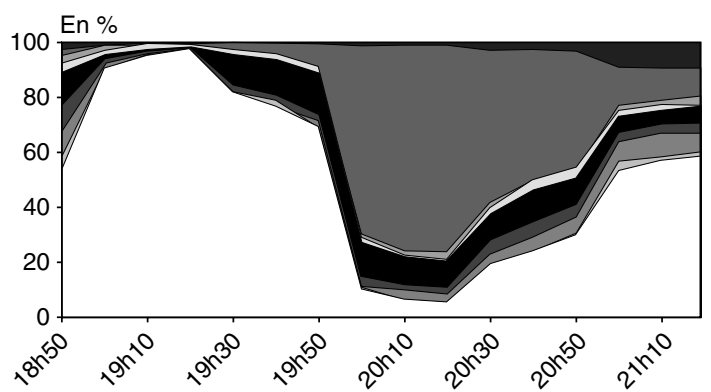

Classe 6

L'alimentation s'insère dans le temps de travail

\section{A. En 1998-1999}

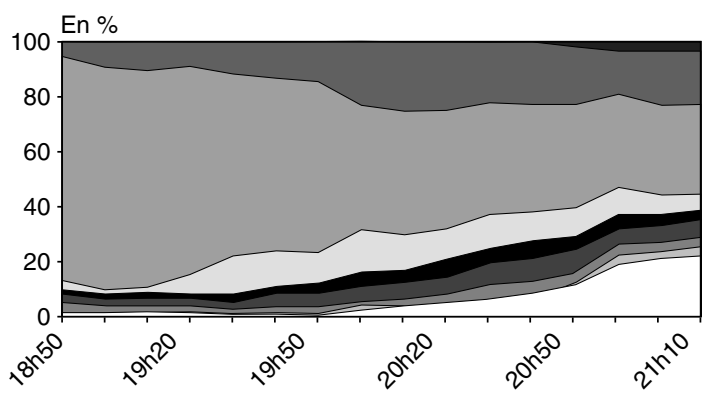

B. En 1985-1986

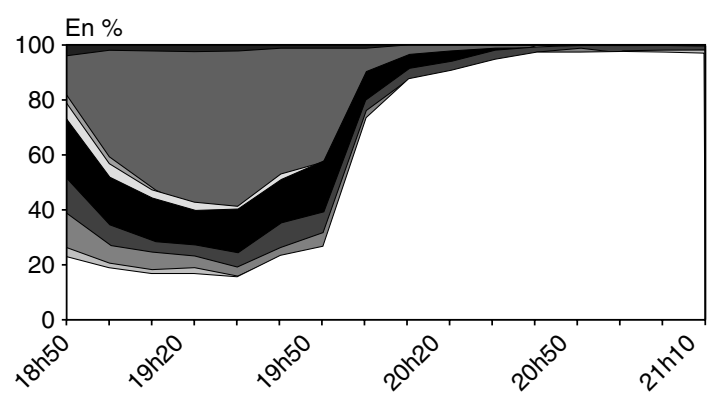

\section{B. En 1985-1986}

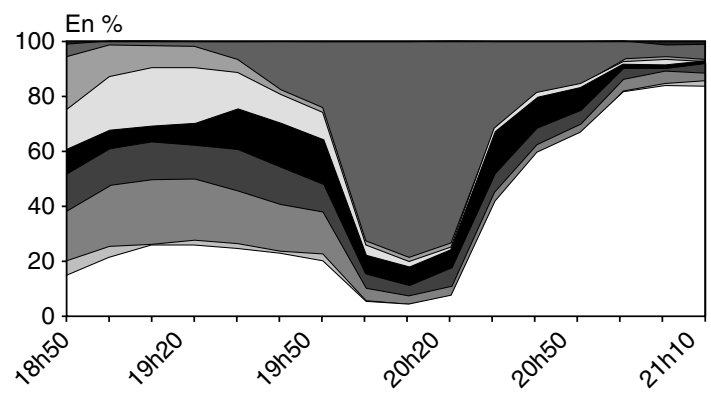

\section{B. En 1985-1986}

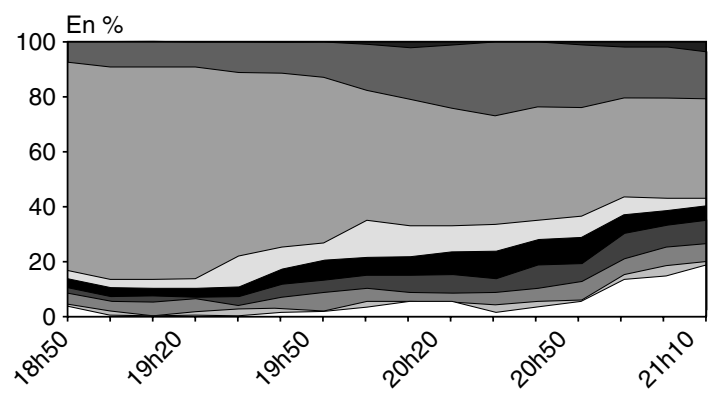

$\square$ Sommeil

$\square$ Repas

$\square$ Travail / Études

$\square$ Trajets

Cuisine / Linge / Ménage

$\square$ Maison / Famille

$\square$ Loisirs (autres que Télévision et Sorties)

$\square$ Rencontres / Spectacles / Promenades

$\square$ Télévision $\square$ Sommeil

$\square$ Repas

$\square$ Travail / Études

$\square$ Trajets

- Cuisine / Linge / Ménage

$\square$ Maison / Famille

$\square$ Loisirs (autres que Télévision et Sorties)

$\square$ Rencontres / Spectacles / Promenades

$\square$ Télévision 
Classe 7

Une prépondérance des plages alimentaires

\section{A. En 1998-1999}

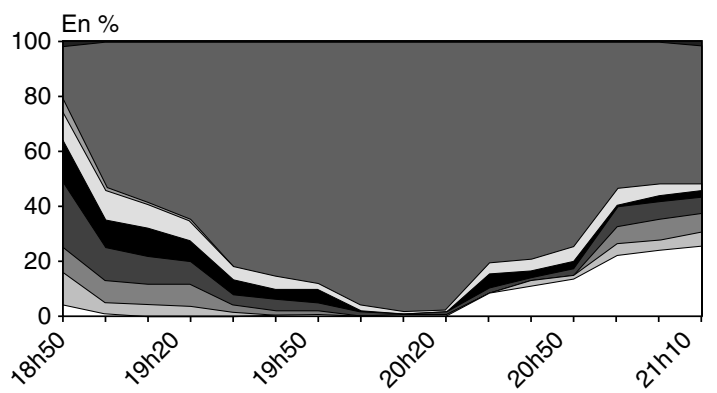

\section{B. En 1985-1986}

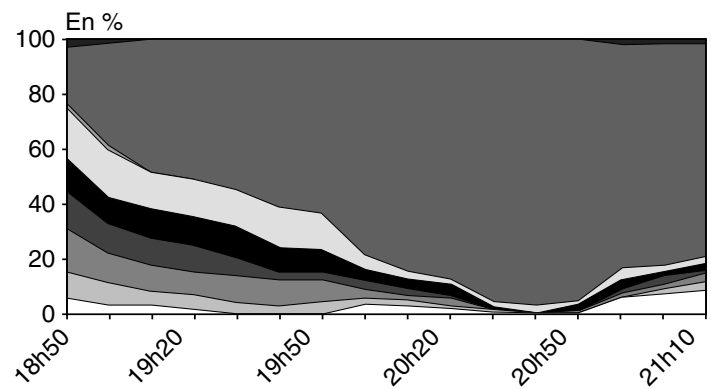

Classe 8

Un repas qui s'insère dans des pratiques de loisirs à l'extérieur

\section{A. En 1998-1999}

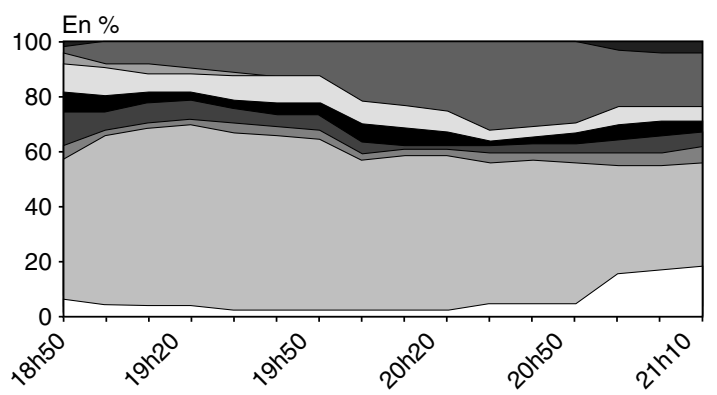

\section{B. En 1985-1986}

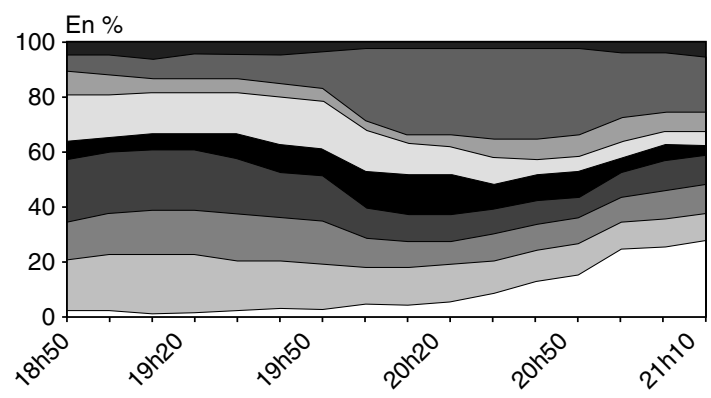

Classe 9

Un repas en seconde moitié de période, après une multitude d'activités qui se prolongent jusqu'à 20h00

\section{A. En 1998-1999}

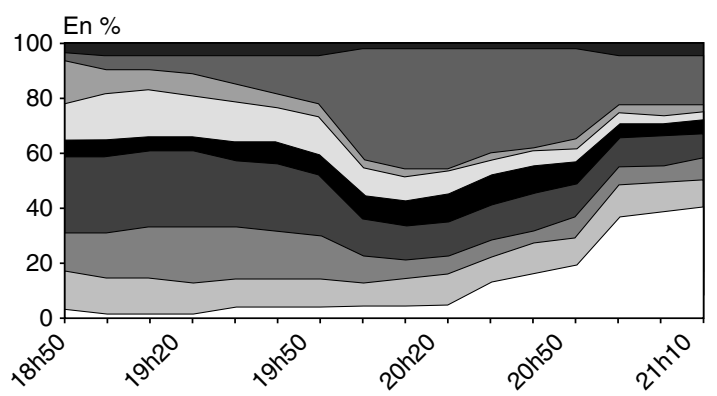

\section{B. En 1985-1986}

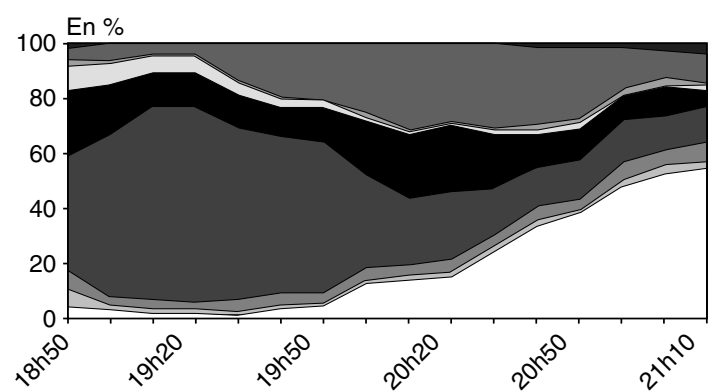

$\square$ Sommeil
$\square$ Repas
$\square$ Travail / Études
$\square$ Trajets
$\square$ Cuisine / Linge / Ménage
$\square$ Maison / Famille
$\square$ Loisirs (autres que Télévision et Sorties)
$\square$ Rencontres / Spectacles / Promenades
$\square$ Télévision

Sommeil

$\square$ Repas

$\square$ Travail / Études

$\square$ Trajets

Cuisine / Linge / Ménage

$\square$ Maison / Famille

$\square$ Loisirs (autres que Télévision et Sorties)

$\square$ Rencontres / Spectacles / Promenades

$\square$ Télévision 
Classe 10

Des individus qui se couchent plus tôt que les autres

\section{A. En 1998-1999}

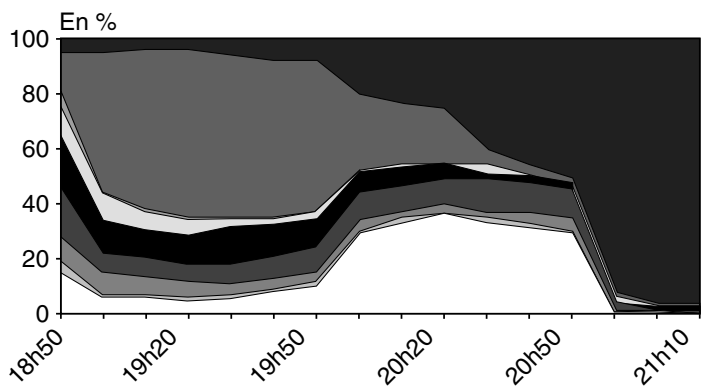

B. En 1985-1986

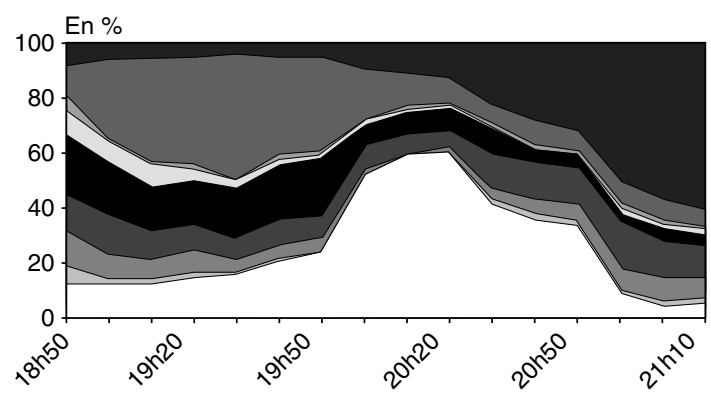

$\square$ Sommeil
$\square$ Repas
$\square$ Travail / Études
$\square$ Trajets
$\square$ Cuisine / Linge / Ménage
$\square$ Maison / Famille
$\square$ Loisirs (autres que Télévision et Sorties)
$\square$ Rencontres / Spectacles / Promenades
$\square$ Télévision

$\square$ Sommeil

$\square$ Repas

$\square$ Travail / Études

$\square$ Trajets

- Cuisine / Linge / Ménage

$\square$ Maison / Famille

$\square$ Loisirs (autres que Télévision et Sorties)

$\square$ Rencontres / Spectacles / Promenades

$\square$ Télévision

Lecture : à 18h50, on lit de bas en haut sur le graphique A de la classe 10 que 15,3\%des individus de 1998 déclarent l'activité " télévision », 5,3\% « rencontres, spectacles, promenades », 7,8\% « autres loisirs d'intérieurs », 17,5\% « maison et famille », 19,7\% « cuisine-Linge-Ménage, 9,7 \% « trajets », 5,3\% « travail-études », 13,8\% une activité de type « repas » et 5,6 \% « sommeil », soit un total de $100 \%$ (cf. annexe A pour le détail des nomenclatures).

Source : enquêtes Emploi du temps 1985-1986 et 1998-1999, Insee. 


\section{L'essentiel du commerce}

$>$ Le portrait des trois principaux secteurs : commerce de détail, commerce et réparation automobiles, commerce de gros.

$>$ Des dossiers thématiques sur le commerce électronique, les marchés et leurs équipements.

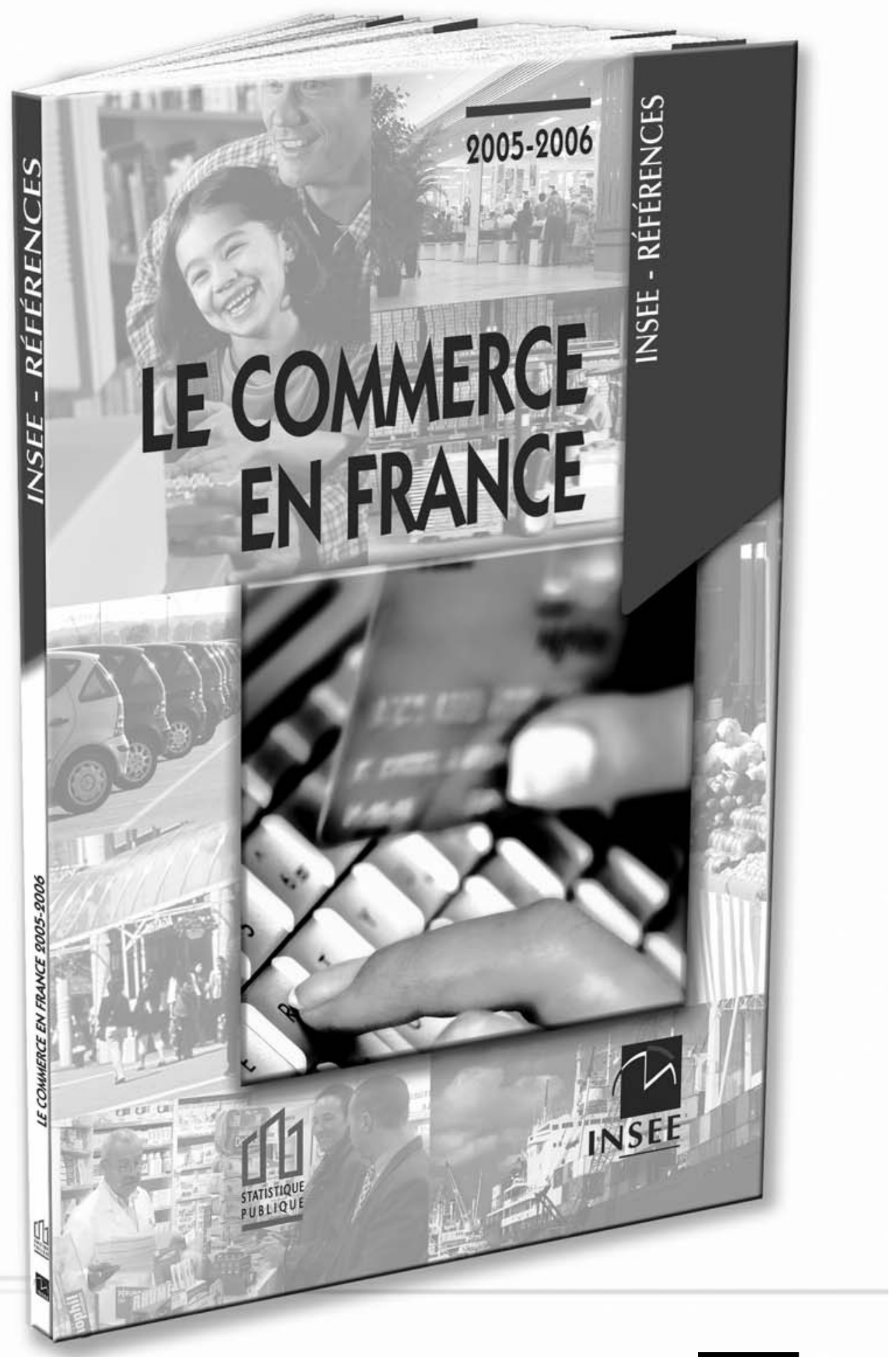

En vente en librairie, à l'Insee et sur www.insee.fr

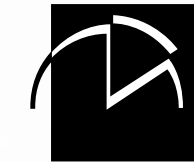

INSEE

$15 €$ - Collection Insee-Références 